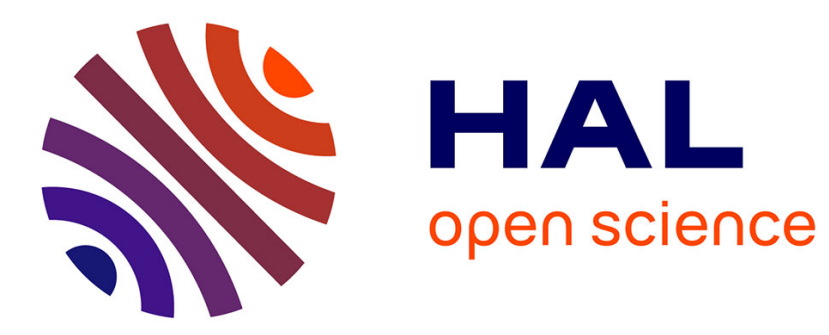

\title{
General sensitivity analysis in data assimilation
}

François-Xavier Le Dimet, Victor P. Shutyaev, Ha Tran Thu

\section{To cite this version:}

François-Xavier Le Dimet, Victor P. Shutyaev, Ha Tran Thu. General sensitivity analysis in data assimilation. Russian Journal of Numerical Analysis and Mathematical Modelling, 2014, 29 (2), pp.107-127. 10.1515/rnam-2014-0009 . hal-00931293

\section{HAL Id: hal-00931293 https://hal.inria.fr/hal-00931293}

Submitted on 15 Jan 2014

HAL is a multi-disciplinary open access archive for the deposit and dissemination of scientific research documents, whether they are published or not. The documents may come from teaching and research institutions in France or abroad, or from public or private research centers.
L'archive ouverte pluridisciplinaire HAL, est destinée au dépôt et à la diffusion de documents scientifiques de niveau recherche, publiés ou non, émanant des établissements d'enseignement et de recherche français ou étrangers, des laboratoires publics ou privés. 


\title{
GENERAL SENSITIVITY ANALYSIS IN DATA ASSIMILATION
}

\author{
F.-X. LE DIMET ${ }^{1}$, V. SHUTYAEV ${ }^{2}$ AND TRAN THU HA ${ }^{3 *}$
}

\begin{abstract}
The problem of variational data assimilation for a nonlinear evolution model is formulated as an optimal control problem to find the initial condition function (analysis). The operator of the model, and hence the optimal solution, depend on the parameters which may contain uncertainties. A response function is considered as a functional of the solution after assimilation. Based on the second-order adjoint techniques, the sensitivity of the response function to the parameters of the model is studied. The gradient of the response function is related to the solution of a non-standard problem involving the coupled system of direct and adjoint equations. The solvability of the non-standard problem is studied. Numerical algorithms for solving the problem are developed. The results are applied for the 2D hydraulic and pollution models. Numerical examples on computation of the gradient of the response function are presented.
\end{abstract}

\section{Statement of the PRoblem}

Consider the mathematical model of a physical process that is described by the evolution problem

$$
\left\{\begin{aligned}
\frac{\partial \varphi}{\partial t} & =F(\varphi, \lambda), \quad t \in(0, T) \\
\left.\varphi\right|_{t=0} & =u
\end{aligned}\right.
$$

where $\varphi=\varphi(t)$ is the unknown function belonging for any $t$ to a Hilbert space $X, u \in X$, $F$ is a nonlinear operator mapping $Y \times Y_{p}$ into $Y$ with $Y=L_{2}(0, T ; X),\|\cdot\|_{Y}=(\cdot, \cdot)_{Y}^{1 / 2}$, $Y_{p}$ is a Hilbert space (the space of parameters of the model). Suppose that for given $u \in X$ and $\lambda \in Y_{p}$ there exists a unique solution $\varphi \in Y$ to (1.1).

Let us introduce the functional

$$
J(u)=\frac{1}{2}\left(V_{1}\left(u-u_{0}\right), u-u_{0}\right)_{X}+\frac{1}{2}\left(V_{2}\left(C \varphi-\varphi_{o b s}\right), C \varphi-\varphi_{o b s}\right)_{Y_{o b s}},
$$

where $u_{0} \in X$ is a prior initial-value function (background state), $\varphi_{o b s} \in Y_{o b s}$ is a prescribed function (observational data), $Y_{o b s}$ is a Hilbert space (observation space), $C: Y \rightarrow Y_{o b s}$ is a

\footnotetext{
*Corresponding author. Email: fxld@yahoo.com

${ }^{1}$ Laboratoire Jean-Kuntzmann, 51 rue des Maths, 38400 Saint Martin d'Hères, France;

${ }^{2}$ Institute of Numerical Mathematics, Russian Academy of Sciences, 119333 Gubkina 8, Moscow, Russia,

${ }^{3}$ Institute of Mechanics - 264 Doi Can,VAST 18 Hoang Quoc Viet and University of Engineering and Technology - VNU,144 Xuan Thuy,

Hanö̈, Vietnam;
} 
linear bounded operator, $V_{1}: X \rightarrow X$ and $V_{2}: Y_{o b s} \rightarrow Y_{o b s}$ are symmetric positive definite operators.

Consider the following data assimilation problem with the aim to identify the initial condition: for given $\lambda \in Y_{p}$ find $u \in X$ and $\varphi \in Y$ such that they satisfy (1.1), and on the set of solutions to (1.1), the functional $J(u)$ takes the minimum value, i.e.

$$
\left\{\begin{aligned}
\frac{\partial \varphi}{\partial t} & =F(\varphi, \lambda), \quad t \in(0, T) \\
\left.\varphi\right|_{t=0} & =u \\
J(u) & =\inf _{v} J(v) .
\end{aligned}\right.
$$

The necessary optimality condition reduces the problem (1.3) to the following optimality system [13]:

$$
\begin{gathered}
\left\{\begin{aligned}
\frac{\partial \varphi}{\partial t} & =F(\varphi, \lambda), \quad t \in(0, T) \\
\left.\varphi\right|_{t=0}= & u
\end{aligned}\right. \\
\left\{\begin{array}{c}
-\frac{\partial \varphi^{*}}{\partial t}-\left(F_{\varphi}^{\prime}(\varphi, \lambda)\right)^{*} \varphi^{*}=-C^{*} V_{2}\left(C \varphi-\varphi_{o b s}\right), \quad t \in(0, T) \\
\left.\varphi^{*}\right|_{t=T}=0, \\
V_{1}\left(u-u_{0}\right)-\left.\varphi^{*}\right|_{t=0}=0
\end{array}\right.
\end{gathered}
$$

with the unknowns $\varphi, \varphi^{*}, u$, where $\left(F_{\varphi}^{\prime}(\varphi, \lambda)\right)^{*}$ is the adjoint to the Frechet derivative of $F$ with respect to $\varphi$, and $C^{*}$ is the adjoint to $C$ defined by $(C \varphi, \psi)_{Y_{o b s}}=\left(\varphi, C^{*} \psi\right)_{Y}, \varphi \in$ $Y, \psi \in Y_{\text {obs }}$.

We assume that the system (1.4)-(1.6) has a unique solution. The system (1.4)-(1.6) may be considered as a generalized model $\mathcal{F}(U, K)=0$ with the state variable $U=\left(\varphi, \varphi^{*}, u\right)$, and it contains all the available information. All the components of $U$ depend on the parameters $\lambda \in Y_{p}$. The purpose of this paper is to study the sensitivity of this generalized model with respect to the parameters.

\section{Sensitivity in the PRESEnCE of DATA}

In the environmental sciences the mathematical models contain parameters which cannot be estimated precisely, because they are used to parametrize some subgrid processes and therefore can not be physically measured. Thus, it is important to be able to estimate the impact of uncertainties on the outputs of the model after assimilation.

Let us introduce a response function $G(\varphi, u, \lambda)$, which is supposed to be a real-valued function and can be considered as a functional on $Y \times X \times Y_{p}$. We are interested in the sensitivity of $G$ with respect to $\lambda$, with $\varphi$ and $u$ obtained from the optimality system (1.4)-(1.6). By definition the sensitivity is defined by the gradient of $G$ with respect to $\lambda$ :

$$
\frac{d G}{d \lambda}=\frac{\partial G}{\partial \varphi} \frac{\partial \varphi}{\partial \lambda}+\frac{\partial G}{\partial u} \frac{\partial u}{\partial \lambda}+\frac{\partial G}{\partial \lambda}
$$


If $\delta \lambda$ is a perturbation on $\lambda$, we get from the optimality system:

$$
\begin{gathered}
\left\{\begin{aligned}
& \frac{\partial \delta \varphi}{\partial t}=F_{\varphi}^{\prime}(\varphi, \lambda) \delta \varphi+F_{\lambda}^{\prime}(\varphi, \lambda) \delta \lambda, \quad t \in(0, T) \\
&\left.\delta \varphi\right|_{t=0}=\delta u
\end{aligned}\right. \\
\left\{\begin{aligned}
-\frac{\partial \delta \varphi^{*}}{\partial t}-\left(F_{\varphi}^{\prime}(\varphi, \lambda)\right)^{*} \delta \varphi^{*}-\left(F_{\varphi \varphi}^{\prime \prime}(\varphi, \lambda) \delta \varphi+F_{\varphi \lambda}^{\prime \prime}(\varphi, \lambda) \delta \lambda\right)^{*} \varphi^{*} & =-C^{*} V_{2} C \delta \varphi \\
\left.\delta \varphi^{*}\right|_{t=T} & =0,
\end{aligned}\right. \\
V_{1} \delta u-\left.\delta \varphi^{*}\right|_{t=0}=0,
\end{gathered}
$$

and

$$
\left(\frac{d G}{d \lambda}, \delta \lambda\right)_{Y_{p}}=\left(\frac{\partial G}{\partial \varphi}, \delta \varphi\right)_{Y}+\left(\frac{\partial G}{\partial u}, \delta u\right)_{X}+\left(\frac{\partial G}{\partial \lambda}, \delta \lambda\right)_{Y_{p}}
$$

where $\delta \varphi, \delta \varphi^{*}$ and $\delta u$ are the Gâteaux derivatives of $\varphi, \varphi^{*}$ and $u$ in the direction $\delta \lambda$ (for example, $\left.\delta \varphi=\frac{\partial \varphi}{\partial \lambda} \delta \lambda\right)$.

To compute the gradient $\nabla_{\lambda} G(\varphi, u, \lambda)$, let us introduce three adjoint variables $P_{1} \in Y$, $P_{2} \in Y$ and $P_{3} \in X$. By taking the inner product of (2.2) by $P_{1},(2.3)$ by $P_{2}$ and of (2.4) by $P_{3}$ and adding them, we obtain:

$$
\begin{gathered}
\left(\delta \varphi,-\frac{\partial P_{1}}{\partial t}-\left(F_{\varphi}^{\prime}(\varphi, \lambda)\right)^{*} P_{1}-\left(F_{\varphi \varphi}^{\prime \prime}(\varphi, \lambda) P_{2}\right)^{*} \varphi^{*}+C^{*} V_{2} C P_{2}\right)_{Y}+\left(\left.\delta \varphi\right|_{t=T},\left.P_{1}\right|_{t=T}\right)_{X}+ \\
+\left(\delta \varphi^{*}, \frac{\partial P_{2}}{\partial t}-F_{\varphi}^{\prime}(\varphi, \lambda) P_{2}\right)_{Y}+\left(\left.\delta \varphi^{*}\right|_{t=0},\left.P_{2}\right|_{t=0}-P_{3}\right)_{X}+ \\
(2.6) \quad+\left(\delta u,-\left.P_{1}\right|_{t=0}+V_{1} P_{3}\right)_{X}+\left(\delta \lambda,-\left(F_{\lambda}^{\prime}(\varphi, \lambda)\right)^{*} P_{1}-\left(F_{\varphi \lambda}^{\prime \prime}(\varphi, \lambda) P_{2}\right)^{*} \varphi^{*}\right)_{Y_{p}}=0 .
\end{gathered}
$$

Here we put

$$
-\frac{\partial P_{1}}{\partial t}-\left(F_{\varphi}^{\prime}(\varphi, \lambda)\right)^{*} P_{1}-\left(F_{\varphi \varphi}^{\prime \prime}(\varphi, \lambda) P_{2}\right)^{*} \varphi^{*}+C^{*} V_{2} C P_{2}=\frac{\partial G}{\partial \varphi},
$$

and

$$
-\left.P_{1}\right|_{t=0}+V_{1} P_{3}=\frac{\partial G}{\partial u},\left.P_{1}\right|_{t=T}=0, \frac{\partial P_{2}}{\partial t}-F_{\varphi}^{\prime}(\varphi, \lambda) P_{2}=0,\left.P_{2}\right|_{t=0}-P_{3}=0 .
$$

Hence, we can exclude the variable $P_{3}$ by

$$
P_{3}=\left.P_{2}\right|_{t=0}
$$

and obtain the initial condition for $P_{2}$ in the form:

$$
\left.V_{1} P_{2}\right|_{t=0}=\frac{\partial G}{\partial u}+\left.P_{1}\right|_{t=0}
$$


Thus, if $P_{1}, P_{2}$ are the solutions of the following system of equations

$$
\left\{\begin{aligned}
-\frac{\partial P_{1}}{\partial t}-\left(F_{\varphi}^{\prime}(\varphi, \lambda)\right)^{*} P_{1}-\left(F_{\varphi \varphi}^{\prime \prime}(\varphi, \lambda) P_{2}\right)^{*} \varphi^{*}+C^{*} V_{2} C P_{2} & =\frac{\partial G}{\partial \varphi}, \quad t \in(0, T) \\
\left.P_{1}\right|_{t=T} & =0
\end{aligned}\right.
$$

$$
\left\{\begin{aligned}
\frac{\partial P_{2}}{\partial t}-F_{\varphi}^{\prime}(\varphi, \lambda) P_{2} & =0, \quad t \in(0, T) \\
\left.V_{1} P_{2}\right|_{t=0} & =\frac{\partial G}{\partial u}+\left.P_{1}\right|_{t=0}
\end{aligned}\right.
$$

then from (2.6) we get

$$
\left(\frac{\partial G}{\partial \varphi}, \delta \varphi\right)_{Y}+\left(\frac{\partial G}{\partial u}, \delta u\right)_{X}=\left(\delta \lambda,\left(F_{\lambda}^{\prime}(\varphi, \lambda)\right)^{*} P_{1}+\left(F_{\varphi \lambda}^{\prime \prime}(\varphi, \lambda) P_{2}\right)^{*} \varphi^{*}\right)_{Y_{p}},
$$

and the gradient of $G$ is given by

$$
\frac{d G}{d \lambda}=\left(F_{\lambda}^{\prime}(\varphi, \lambda)\right)^{*} P_{1}+\left(F_{\varphi \lambda}^{\prime \prime}(\varphi, \lambda) P_{2}\right)^{*} \varphi^{*}+\frac{\partial G}{\partial \lambda} .
$$

We get a coupled system of two differential equations (2.7) and (2.8) of the first order with respect to time. One equation has a final condition (backward problem) while the other has an initial condition (forward problem) depending on the initial value for the first equation: that is a non-standard problem.

\section{Solving the NON-STANDARD PROBlem: A Method BASED On OPtimal CONTROL}

The method proposed is based on the theory of optimal control [13]. We consider the system (2.7)-(2.8) in the form

$$
\begin{aligned}
& \left\{\begin{aligned}
-\frac{\partial P_{1}}{\partial t}+A^{*} P_{1}+B P_{2} & =f, \quad t \in(0, T) \\
\left.P_{1}\right|_{t=T} & =0,
\end{aligned}\right. \\
& \left\{\begin{aligned}
\frac{\partial P_{2}}{\partial t}+A P_{2} & =0, \quad t \in(0, T) \\
\left.V_{1} P_{2}\right|_{t=0} & =\left.P_{1}\right|_{t=0}+g
\end{aligned}\right.
\end{aligned}
$$

where $A=-F_{\varphi}^{\prime}(\varphi, \lambda), B=-\left(F_{\varphi \varphi}^{\prime \prime}(\varphi, \lambda) \cdot\right)^{*} \varphi^{*}+C^{*} V_{2} C$ are linear operators mapping $Y$ into $Y, f=\frac{\partial G}{\partial \varphi} \in Y, g=\frac{\partial G}{\partial u} \in X$. 
Let transform (3.1)-(3.2) into a problem of optimal control. Instead of (3.2) we consider the problem

$$
\left\{\begin{aligned}
\frac{\partial P_{2}}{\partial t}+A P_{2} & =0, \quad t \in(0, T) \\
\left.P_{2}\right|_{t=0} & =v
\end{aligned}\right.
$$

with some initial condition $v \in X$. We assume that for given $f \in Y, v \in X$ the coupled problem (3.1), (3.3) has a unique solution $P_{1}, P_{2}$ for $t \in[0, T]$. Let $P_{1}(0, U)$ be the value of $P_{1}$ at time $t=0$ for the value $v$ of $\left.P_{2}\right|_{t=0}$. We define the cost function

$$
J_{P}(v)=\frac{1}{2}\left\|V_{1} v-P_{1}(0, v)-g\right\|_{X}^{2} .
$$

The problem becomes the determination of $v^{*}$ by minimizing $J_{P}$. We can expect that at the optimum, $V_{1} v-P_{1}(0, v)-g=0$ and the problem will be solved. The procedure is similar to the one used in section 2 .

Let $\delta v$ be a perturbation on $v$, then from (3.1), (3.3), (3.4) we get

$$
\begin{gathered}
\left\{\begin{array}{r}
-\frac{\partial \delta P_{1}}{\partial t}+A^{*} \delta P_{1}+B \delta P_{2}=0, \quad t \in(0, T) \\
\left.\delta P_{1}\right|_{t=T}=0
\end{array}\right. \\
\left\{\begin{array}{r}
\frac{\partial \delta P_{2}}{\partial t}+A \delta P_{2}=0, \quad t \in(0, T) \\
\left.\delta P_{2}\right|_{t=0}=\delta v,
\end{array}\right. \\
J_{P}^{\prime}(v) \delta v=\left(V_{1} v-\left.P_{1}\right|_{t=0}-g, V_{1} \delta v-\left.\delta P_{1}\right|_{t=0}\right)_{X},
\end{gathered}
$$

where $\delta P_{1}, \delta P_{2}$ are the Gâteaux derivatives of $P_{1}, P_{2}$ with respect to $v$ in the direction $\delta v$.

To compute the gradient $\nabla J_{P}(v)$ let us introduce the adjoint variables $Q_{1}, Q_{2} \in Y$. By taking the inner product of (3.5) by $Q_{1}$ and (3.6) by $Q_{2}$, we obtain

$$
\begin{gathered}
\left(\delta P_{1}, \frac{\partial Q_{1}}{\partial t}+A Q_{1}\right)_{Y}+\left(\left.\delta P_{1}\right|_{t=0},\left.Q_{1}\right|_{t=0}\right)_{X}+ \\
+\left(\delta P_{2},-\frac{\partial Q_{2}}{\partial t}+A^{*} Q_{2}+B Q_{1}\right)_{Y}+\left(\left.\delta P_{2}\right|_{t=T},\left.Q_{2}\right|_{t=T}\right)_{X}-\left(\delta v,\left.Q_{2}\right|_{t=0}\right)_{X}=0 .
\end{gathered}
$$

If $Q_{1}$ and $Q_{2}$ are defined as the solution of the system

$$
\left\{\begin{aligned}
\frac{\partial Q_{1}}{\partial t}+A Q_{1} & =0, \quad t \in(0, T) \\
\left.Q_{1}\right|_{t=0} & =V_{1} v-\left.P_{1}\right|_{t=0}-g,
\end{aligned}\right.
$$




$$
\left\{\begin{aligned}
-\frac{\partial Q_{2}}{\partial t}+A^{*} Q_{2}+B Q_{1} & =0, \quad t \in(0, T) \\
\left.Q_{2}\right|_{t=T} & =0
\end{aligned}\right.
$$

then (3.8) implies $\left(\left.\delta P_{1}\right|_{t=0}, V_{1} v-\left.P_{1}\right|_{t=0}-g\right)_{X}=\left(\delta v,\left.Q_{2}\right|_{t=0}\right)_{X}$, and we get for the gradient:

$$
\nabla J_{P}(v)=V_{1}\left(V_{1} v-\left.P_{1}\right|_{t=0}-g\right)-\left.Q_{2}\right|_{t=0} .
$$

\section{Control equation via Hessian}

The nesessary optimality condition reduces the non-standard problem to the optimality system:

$$
\begin{aligned}
& \left\{\begin{aligned}
-\frac{\partial P_{1}}{\partial t}+A^{*} P_{1}+B P_{2} & =f, \quad t \in(0, T) \\
\left.P_{1}\right|_{t=T} & =0,
\end{aligned}\right. \\
& \left\{\begin{aligned}
\frac{\partial P_{2}}{\partial t}+A P_{2} & =0, \quad t \in(0, T) \\
\left.P_{2}\right|_{t=0} & =v
\end{aligned}\right. \\
& \left\{\begin{aligned}
\frac{\partial Q_{1}}{\partial t}+A Q_{1} & =0, \quad t \in(0, T) \\
\left.Q_{1}\right|_{t=0} & =V_{1} v-\left.P_{1}\right|_{t=0}-g
\end{aligned}\right. \\
& \left\{\begin{aligned}
-\frac{\partial Q_{2}}{\partial t}+A^{*} Q_{2}+B Q_{1} & =0, \quad t \in(0, T) \\
\left.Q_{2}\right|_{t=T} & =0
\end{aligned}\right. \\
& V_{1}\left(V_{1} v-\left.P_{1}\right|_{t=0}-g\right)-\left.Q_{2}\right|_{t=0}=0
\end{aligned}
$$

with the unknowns $v \in X, P_{1}, P_{2}, Q_{1}, Q_{2} \in Y$.

The system (4.1)-(4.5) is equivalent to a single equation for $v$ (the control equation):

$$
H v=F,
$$

where $H$ is the Hessian of the functional $J_{P}$, defined on $w \in X$ by the successive solutions of the following problems:

$$
\left\{\begin{aligned}
\frac{\partial \hat{P}_{2}}{\partial t}+A \hat{P}_{2} & =0, \quad t \in(0, T) \\
\left.\hat{P}_{2}\right|_{t=0} & =w
\end{aligned}\right.
$$




$$
\begin{gathered}
\left\{\begin{array}{c}
-\frac{\partial \hat{P}_{1}}{\partial t}+A^{*} \hat{P}_{1}+B \hat{P}_{2}=0, \quad t \in(0, T) \\
\left.P_{1}\right|_{t=T}=0,
\end{array}\right. \\
\left\{\begin{array}{r}
\frac{\partial \hat{Q}_{1}}{\partial t}+A \hat{Q}_{1}=0, \quad t \in(0, T) \\
\left.\hat{Q}_{1}\right|_{t=0}=V_{1} w-\left.\hat{P}_{1}\right|_{t=0},
\end{array}\right. \\
\left\{\begin{array}{c}
-\frac{\partial \hat{Q}_{2}}{\partial t}+A^{*} \hat{Q}_{2}+B \hat{Q}_{1}=0, \quad t \in(0, T) \\
\left.\hat{Q}_{2}\right|_{t=T}=0, \\
H w=V_{1}\left(V_{1} w-\left.\hat{P}_{1}\right|_{t=0}\right)-\left.\hat{Q}_{2}\right|_{t=0}=0,
\end{array}\right.
\end{gathered}
$$

and the right-hand side $F$ is defined by the successive solutions of the following problems:

$$
\begin{aligned}
& \left\{\begin{aligned}
-\frac{\partial \tilde{P}_{1}}{\partial t}+A^{*} \tilde{P}_{1} & =f, \quad t \in(0, T) \\
\left.\tilde{P}_{1}\right|_{t=T} & =0,
\end{aligned}\right. \\
& \left\{\begin{aligned}
\frac{\partial \tilde{Q}_{1}}{\partial t}+A \tilde{Q}_{1} & =0, \quad t \in(0, T) \\
\left.\tilde{Q}_{1}\right|_{t=0} & =-\left.\tilde{P}_{1}\right|_{t=0}-g,
\end{aligned}\right. \\
& \left\{\begin{aligned}
-\frac{\partial \tilde{Q}_{2}}{\partial t}+A^{*} \tilde{Q}_{2}+B \tilde{Q}_{1} & =0, \quad t \in(0, T) \\
\left.\tilde{Q}_{2}\right|_{t=T} & =0
\end{aligned}\right. \\
& F=V_{1}\left(\left.\tilde{P}_{1}\right|_{t=0}+g\right)+\left.\tilde{Q}_{2}\right|_{t=0} .
\end{aligned}
$$

The Hessian $H$ maps $X$ into $X$, it is symmetric and

$$
(H w, w)_{X}=\left\|V_{1} w-\left.\hat{P}_{1}\right|_{t=0}\right\|_{X}^{2},
$$

where $\hat{P}_{1}$ is the solution to (4.8). Indeed, since

$$
(H w, w)_{X}=\left(V_{1}^{2} w, w\right)_{X}-\left(\left.V_{1} \hat{P}_{1}\right|_{t=0}, w\right)_{X}-\left(\left.\hat{Q}_{2}\right|_{t=0}, w\right)_{X},
$$

and

$$
\begin{gathered}
\left(\left.\hat{Q}_{2}\right|_{t=0}, w\right)_{X}=\left(\left.\hat{Q}_{2}\right|_{t=0},\left.\hat{P}_{2}\right|_{t=0}\right)_{X}=-\left(B \hat{Q}_{1}, \hat{P}_{2}\right)_{Y}=-\left(\hat{Q}_{1}, B \hat{P}_{2}\right)_{Y}= \\
=\left(0, \hat{P}_{1}\right)_{Y}+\left(\left.\hat{P}_{1}\right|_{t=0},\left.\hat{Q}_{1}\right|_{t=0}\right)_{X}=\left(V_{1} w-\left.\hat{P}_{1}\right|_{t=0},\left.\hat{P}_{1}\right|_{t=0}\right)_{X},
\end{gathered}
$$

then

$$
(H w, w)_{X}=\left(V_{1}^{2} w, w\right)_{X}-\left(\left.V_{1} \hat{P}_{1}\right|_{t=0}, w\right)_{X}-\left(V_{1} w-\left.\hat{P}_{1}\right|_{t=0},\left.\hat{P}_{1}\right|_{t=0}\right)_{X}=\left\|V_{1} w-\left.\hat{P}_{1}\right|_{t=0}\right\|_{X}^{2} .
$$


Moreover, using the definitions of the operators $A$ and $B$, it is easily seen that

$$
V_{1} w-\left.\hat{P}_{1}\right|_{t=0}=\mathcal{H} w
$$

where $\mathcal{H}$ is the Hessian of the original functional $J$. Then, under the assumption that $\mathcal{H}$ is positive definite, we obtain

$$
(H w, w)_{X}=(\mathcal{H} w, \mathcal{H} w)_{X} \geq c\|w\|_{X}^{2} .
$$

where $c=\lambda_{\text {min }}^{2}(\mathcal{H})$, and $\lambda_{\min }(\mathcal{H})$ is the lower spectrum bound of the operator $\mathcal{H}$.

Thus, the Hessian $H$ is symmetric and positive definite, and therefore, the control equation (4.6) is correctly and everywhere solvable [21], i.e. for every $F \in X$ there exists a unique solution $v \in X$ of (4.6) and the estimate holds:

$$
\|v\|_{X} \leq c_{1}\|F\|_{X}, \quad c_{1}=\text { const }>0 .
$$

Therefore, we have proved that the non-standard optimal control problem with the functional (3.4) has a unique solution.

\section{A SECOND Method to SOlVE The NON-STANDARD PROBlem}

Let us return to the non-standard problem (2.7)-(2.8) and rewrite it in an equivalent form:

$$
\begin{aligned}
& \left\{\begin{aligned}
-\frac{\partial P_{1}}{\partial t}-\left(F_{\varphi}^{\prime}(\varphi, \lambda)\right)^{*} P_{1}-\left(F_{\varphi \varphi}^{\prime \prime}(\varphi, \lambda) P_{2}\right)^{*} \varphi^{*}+C^{*} V_{2} C P_{2} & =\frac{\partial G}{\partial \varphi}, \quad t \in(0, T) \\
\left.P_{1}\right|_{t=T} & =0
\end{aligned}\right. \\
& \left\{\begin{aligned}
\frac{\partial P_{2}}{\partial t}-F_{\varphi}^{\prime}(\varphi, \lambda) P_{2} & =0, \quad t \in(0, T) \\
\left.P_{2}\right|_{t=0} & =v
\end{aligned}\right. \\
& V_{1} v-\left.P_{1}\right|_{t=0}=\frac{\partial G}{\partial u} .
\end{aligned}
$$

Here we have three unknowns: $v \in X, P_{1}, P_{2} \in Y$. Let us write (5.1)-(5.3) in the form of an operator eqution for $v$. We define the operator $\mathcal{H}$ by the successive solution of the following problems:

$$
\begin{aligned}
& \left\{\begin{aligned}
\frac{\partial \phi}{\partial t}-F_{\varphi}^{\prime}(\varphi, \lambda) \phi & =0, \quad t \in(0, T) \\
\left.\phi\right|_{t=0} & =w
\end{aligned}\right. \\
& \left\{\begin{aligned}
-\frac{\partial \phi^{*}}{\partial t}-\left(F_{\varphi}^{\prime}(\varphi, \lambda)\right)^{*} \phi^{*}-\left(F_{\varphi \varphi}^{\prime \prime}(\varphi, \lambda) \phi\right)^{*} \varphi^{*} & =-C^{*} V_{2} C \phi, \quad t \in(0, T) \\
\left.\phi^{*}\right|_{t=T} & =0,
\end{aligned}\right.
\end{aligned}
$$




$$
\mathcal{H} w=V_{1} w-\left.\phi^{*}\right|_{t=0} .
$$

Then (5.1)-(5.3) is equivalent to the following equation in $X$ :

$$
\mathcal{H} v=\mathcal{F}
$$

with the right-hand side $\mathcal{F}$ defined by

$$
\mathcal{F}=\frac{\partial G}{\partial u}+\left.\tilde{\phi}^{*}\right|_{t=0}
$$

where $\tilde{\phi}^{*}$ is the solution to the adjoint problem:

$$
\left\{\begin{aligned}
-\frac{\partial \tilde{\phi}^{*}}{\partial t}-\left(F_{\varphi}^{\prime}(\varphi, \lambda)\right)^{*} \tilde{\phi}^{*} & =\frac{\partial G}{\partial \varphi}, \quad t \in(0, T) \\
\left.\tilde{\phi}^{*}\right|_{t=T} & =0 .
\end{aligned}\right.
$$

It is easily seen that the operator $\mathcal{H}$ defined by (5.4)-(5.6) is the Hessian of the original functional $J$ considered on the optimal solution $u$ of the problem $(1.4)-(1.6): J^{\prime \prime}(u)=\mathcal{H}$. Under the assumption that $\mathcal{H}$ is positive definite, the operator equation (5.7) is correctly and everywhere solvable in $X$, i.e. for every $\mathcal{F}$ there exists a unique solution $v \in X$ and

$$
\|v\|_{X} \leq c\|\mathcal{H}\|_{X}, \quad c=\text { const }>0 .
$$

Therefore, under the assumption that $J^{\prime \prime}(u)$ is positive definite on the optimal solution, the non-standard problem (2.7)-(2.8) has a unique solution $P_{1}, P_{2} \in Y$.

Based on the above consideration, we can formulate the following algorithm to solve the non-standard problem:

1) For $\frac{\partial G}{\partial u} \in X, \frac{\partial G}{\partial \varphi} \in Y$ solve the adjoint problem

$$
\left\{\begin{aligned}
-\frac{\partial \tilde{\phi}^{*}}{\partial t}-\left(F_{\varphi}^{\prime}(\varphi, \lambda)\right)^{*} \tilde{\phi}^{*} & =\frac{\partial G}{\partial \varphi}, \quad t \in(0, T) \\
\left.\tilde{\phi}^{*}\right|_{t=T} & =0
\end{aligned}\right.
$$

and put

$$
\mathcal{F}=\frac{\partial G}{\partial u}+\left.\tilde{\phi}^{*}\right|_{t=0}
$$

2) Find $v$ by solving

$$
\mathcal{H} v=\mathcal{F}
$$

with the Hessian of the original functional $J$ defined by (5.4)-(5.6).

3) Solve successively the direct and adjoint problems

$$
\left\{\begin{aligned}
\frac{\partial P_{2}}{\partial t}-F_{\varphi}^{\prime}(\varphi, \lambda) P_{2} & =0, \quad t \in(0, T) \\
\left.P_{2}\right|_{t=0} & =v,
\end{aligned}\right.
$$




$$
\left\{\begin{aligned}
-\frac{\partial \tilde{P}_{1}}{\partial t}-\left(F_{\varphi}^{\prime}(\varphi, \lambda)\right)^{*} \tilde{P}_{1}-\left(F_{\varphi \varphi}^{\prime \prime}(\varphi, \lambda) P_{2}\right)^{*} \varphi^{*}+C^{*} V_{2} C P_{2} & =0, \quad t \in(0, T) \\
\left.\tilde{P}_{1}\right|_{t=T} & =0
\end{aligned}\right.
$$

and put

$$
P_{1}=\tilde{P}_{1}+\tilde{\phi}^{*}
$$

Thus, we obtain $P_{1}, P_{2} \in Y$ as the solutions to the non-standard problem (2.7)-(2.8).

Remark 1. In the above consideration, we have assumed that the direct and adjoint tangent linear problems of the form

$$
\begin{gathered}
\left\{\begin{aligned}
\frac{\partial \phi}{\partial t}-F_{\varphi}^{\prime}(\varphi, \lambda) \phi & =f, \quad t \in(0, T) \\
\left.\phi\right|_{t=0} & =w,
\end{aligned}\right. \\
\left\{\begin{aligned}
-\frac{\partial \phi^{*}}{\partial t}-\left(F_{\varphi}^{\prime}(\varphi, \lambda)\right)^{*} \phi^{*} & =g, \quad t \in(0, T) \\
\left.\phi^{*}\right|_{t=T} & =0
\end{aligned}\right.
\end{gathered}
$$

with $w \in X, f, g \in Y$ have the unique solutions $\phi, \phi^{*} \in Y$ with $\left.\phi\right|_{t=T},\left.\phi^{*}\right|_{t=0} \in X$.

Based on the presented theory, in the forthcoming sections we consider an application to the 2D hydraulic and pollution models.

\section{Mathematical formulation of the 2D WATER Pollution Problem}

In this part 2D hydraulic and pollution models are used to describe the transport of the pollution substances. The $2 \mathrm{D}$ pollution water model consists of a hydraulic model and a transport-diffusion model of pollution substances. In the hydraulic model the Saint-Venant equations are used [23]:

$$
\begin{gathered}
\frac{\partial h}{\partial t}+\frac{\partial(u h)}{\partial x}+\frac{\partial(v h)}{\partial y}=0, \quad \text { in } \Omega, \\
\frac{\partial u}{\partial t}+u \frac{\partial u}{\partial x}+v \frac{\partial u}{\partial y}+g \frac{\partial h}{\partial x}=-\frac{g u\left(u^{2}+v^{2}\right)^{1 / 2}}{K_{x}^{2} h^{4 / 3}}-g \frac{\partial z_{b}}{\partial x}, \quad \text { in } \Omega, \\
\frac{\partial v}{\partial t}+u \frac{\partial v}{\partial x}+v \frac{\partial v}{\partial y}+g \frac{\partial h}{\partial y}=-\frac{g v\left(u^{2}+v^{2}\right)^{1 / 2}}{K_{y}^{2} h^{4 / 3}}-g \frac{\partial z_{b}}{\partial y}, \quad \text { in } \Omega
\end{gathered}
$$

where $\Omega$ is a bounded domain of $R^{2}$ with the boundary $\Gamma, z_{b}$ is the bottom elevation, $h=z-z_{b}$ is the water depth, and $z$ is the free surface elevation, $u$ is the average velocity in the $x$ direction, $v$ is the average velocity in the $y$ direction, $g$ is the gravity acceleration, $K_{x}$ and $K_{y}$ are the Strickler coefficients in the $x$ and $y$ directions, respectively. 
We suppose that a substance is dissolved in water. Then the transport and diffusion processes of pollution substances are described by the following equation [24]:

$$
\frac{\partial C}{\partial t}+u \frac{\partial C}{\partial x}+v \frac{\partial C}{\partial y}-\eta \Delta C=K C+S, \quad \text { in } \Omega,
$$

where $\Delta=\left(\frac{\partial^{2}}{\partial x^{2}}+\frac{\partial^{2}}{\partial y^{2}}\right), C=C(x, y, t)$ is the concentration of the substance, $K$ is the conversion coefficient, $S=S(x, y)$ is the pollution function source in fluid, $\eta$ is the diffusion coefficient.

For $X=(h, u, v)^{T}$ and $C$ we have the initial conditions:

$$
\left.X\right|_{t=0}=(h(x, y, 0), u(x, y, 0), v(x, y, 0))^{T}=U, C(x, y, 0)=V .
$$

The boundary conditions are: $\mathbf{U} \cdot \vec{n}=\overline{\mathbf{U}}_{i n}(t), C(x, y, t) \vec{n}=\bar{C}_{i n}(t)$ on the inflow boundary $\Gamma_{1} ; h(x, y, t)=\bar{h}(t), \frac{\partial C}{\partial \vec{n}}=0$ on the outflow boundary $\Gamma_{2} ; \mathbf{U} \cdot \vec{n}=0, \frac{\partial C}{\partial \vec{n}}=0$ on the solid wall $S_{\mathrm{w}}$, where $\mathbf{U}=(u(x, y, t), v(x, y, t)), \Gamma=\Gamma_{1} \cup \Gamma_{2} \cup S_{\mathrm{w}}$ is the boundary of the domain $\Omega, \vec{n}=\left(n_{x}, n_{y}\right)$ is the unit normal vector to $\Gamma$.

Equations (6.1)-(6.4) with boundary and initial conditions are rewritten as follows:

$$
\begin{aligned}
& \left\{\begin{aligned}
\frac{\partial X}{\partial t}+\frac{\partial \mathbf{A}(X)}{\partial x}+\frac{\partial \mathbf{B}(X)}{\partial y} & =F(X), \quad \text { in } \Omega \\
n_{x} u+n_{y} v & =\overline{\mathbf{U}}_{i n}, \quad \text { on } \Gamma_{1} \\
n_{x} u+n_{y} v & =0, \quad \text { on } S_{W} \\
h & =\bar{h}(t), \quad \text { on } \Gamma_{2}, \\
X(0) & =U,
\end{aligned}\right. \\
& \left\{\begin{aligned}
\frac{\partial C}{\partial t}+u \frac{\partial C}{\partial x}+v \frac{\partial C}{\partial y}-\eta \triangle C & =K C+S, \quad \text { in } \Omega \\
C & =\bar{C}_{i n}, \quad \text { on } \Gamma_{1}, \\
\frac{\partial C}{\partial \vec{n}} & =0, \quad \text { on } \Gamma_{2} \bigcup S_{W}, \\
C(0) & =V,
\end{aligned}\right.
\end{aligned}
$$

where:

$$
\begin{gathered}
\mathbf{A}(X)=\left(\begin{array}{c}
u h \\
\frac{1}{2} u^{2}+g h \\
u v
\end{array}\right), \quad \mathbf{B}(X)=\left(\begin{array}{c}
v h \\
u v \\
\frac{1}{2} v^{2}+g h
\end{array}\right), \\
\quad F(X)=\left(\begin{array}{c}
0 \\
-g u \frac{\sqrt{u^{2}+v^{2}}}{K_{x}^{2} h^{4 / 3}}+u \frac{\partial v}{\partial y}-g \frac{\partial z_{b}}{\partial x} \\
-g v \frac{\sqrt{u^{2}+v^{2}}}{K_{y}^{2} h^{4 / 3}}+v \frac{\partial u}{\partial x}-g \frac{\partial z_{b}}{\partial y}
\end{array}\right) .
\end{gathered}
$$




\section{VARIATIONAL DATA ASSIMILATION PROBLEM}

According to (1.2), we define the cost function $J$ by

$$
J(U, V)=\frac{1}{2}\left(V_{1 X}\left(U-X_{0}\right),\left(U-X_{0}\right)\right)_{X_{X}}+\frac{1}{2}\left(V_{1 C}\left(V-C_{0}\right),\left(V-C_{0}\right)\right)_{X_{C}}
$$

$+\frac{1}{2}\left(V_{2 X}\left(H_{X} X-X_{o b s}\right),\left(H_{X} X-X_{o b s}\right)\right)_{Y_{X o b s}}+\frac{1}{2}\left(V_{2 C}\left(H_{c} C-C_{o b s}\right),\left(H_{c} C-C_{o b s}\right)\right)_{Y_{C o b s}}$,

where $X_{C}=L_{2}(\Omega), X_{X}=L_{2}(\Omega) \times L_{2}(\Omega) \times L_{2}(\Omega), Y_{C}=L_{2}\left(0, T ; X_{C}\right), Y_{X}=L_{2}\left(0, T ; X_{X}\right)$, $(X, C) \in Y_{X} \times Y_{C} ;(U, V) \in X_{X} \times X_{C}, X_{0}, C_{0} \in X_{X} \times X_{C}$ is a prior initial-value function (background state), $\left(X_{o b s}, C_{o b s}\right) \in Y_{X o b s} \times Y_{C o b s}$ is a prescribed function (observational data), $Y_{X o b s}, Y_{C o b s}$ are Hilbert spaces (observation spaces), $H_{X}: Y_{X} \rightarrow Y_{X o b s}, H_{c}: Y_{C} \rightarrow$ $Y_{C o b s}$ are linear bounded operators, $V_{1 X}: X_{X} \rightarrow X_{X}, V_{1 C}: X_{C} \rightarrow X_{C}, V_{2 X}: Y_{X o b s} \rightarrow$ $Y_{X o b s}, V_{2 C}: Y_{C o b s} \rightarrow Y_{C o b s}$ are symmetric positive definite operators.

Consider the following data assimilation problem with the aim to identify the initial condition: for given $S$ find $U=X(0) \in X_{X}, V=C(0) \in X_{C}, X \in Y_{X}$ and $C \in Y_{C}$ such that they satisfy (6.5)-(6.6), and on the set of solutions to (6.5)-(6.6), the functional $J(U, V)$ takes the minimum value.

Following section 1, the data assimilation problem is written in the form:

$$
\left\{\begin{aligned}
\frac{\partial X}{\partial t}+\frac{\partial \mathbf{A}(X)}{\partial x}+\frac{\partial \mathbf{B}(X)}{\partial y} & =F(X), \quad \text { in } \Omega, \\
\frac{\partial C}{\partial t}+u \frac{\partial C}{\partial x}+v \frac{\partial C}{\partial y}-\eta \triangle C & =K C+S, \quad \text { in } \Omega, \\
n_{x} u+n_{y} v & =\overline{\mathbf{U}}_{i n}, \quad \text { on } \Gamma_{1}, \\
n_{x} u+n_{y} v & =0, \quad \text { on } S_{W}, \\
h & =\bar{h}(t), \quad \text { on } \Gamma_{2}, \\
C & =\bar{C}_{i n}, \quad \text { on } \Gamma_{1} \\
\frac{\partial C}{\partial \vec{n}} & =0, \quad \text { on } \Gamma_{2} \bigcup S_{W}, \\
C(0) & =V \\
X(0) & =U \\
J(U, V) & =\inf _{U^{*}, V^{*}} J\left(U^{*}, V^{*}\right) .
\end{aligned}\right.
$$


According to (1.4)-(1.6), the necessary optimality condition reduces problem (7.2) to the following optimality system:

$$
\left\{\begin{aligned}
\frac{\partial X}{\partial t}+\frac{\partial \mathbf{A}(X)}{\partial x}+\frac{\partial \mathbf{B}(X)}{\partial y} & =F(X), \quad \text { in } \Omega, \\
\frac{\partial C}{\partial t}+u \frac{\partial C}{\partial x}+v \frac{\partial C}{\partial y}-\eta \triangle C & =K C+S, \quad \text { in } \Omega, \\
n_{x} u+n_{y} v & =\overline{\mathbf{U}}_{i n}, \quad \text { on } \Gamma_{1}, \\
n_{x} u+n_{y} v & =0, \quad \text { on } S_{W}, \\
h & =\bar{h}(t), \quad \text { on } \Gamma_{2}, \\
C & =\bar{C}_{i n}, \quad \text { on } \Gamma_{1} \\
\frac{\partial C}{\partial \vec{n}} & =0, \quad \text { on } \Gamma_{2} \bigcup S_{W}, \\
C(0) & =V, \\
X(0) & =U,
\end{aligned}\right.
$$

$$
\begin{aligned}
& \begin{aligned}
\frac{\partial P}{\partial t} & =\frac{\partial A^{*}(X, P)}{\partial x}+\frac{\partial B^{*}(X, P)}{\partial y}-F^{*}(X, P)+F_{0}(Q, C)+H_{X}^{*} V_{2 X}\left(H_{X} X-X_{o b s}\right) \\
P_{2} n_{x}+P_{3} n_{y} & =0 \quad \text { on } S_{W}
\end{aligned} \\
& P_{2}=-\frac{\mathbf{U}_{i n}}{g} P_{1} n_{x} \quad \text { on } \Gamma_{1} \text {, } \\
& P_{3}=-\frac{\mathbf{U}_{i n}}{g} P_{1} n_{y} \quad \text { on } \Gamma_{1} \\
& P_{2}=-\frac{h p_{1} n_{x}}{\mathrm{U} \vec{n}} \text { on } \Gamma_{2} \text {, } \\
& P_{3}=-\frac{h P_{1} n_{y}}{\mathbf{U} \vec{n}} \text { on } \Gamma_{2} \text {, } \\
& P(T)=0 \text {, }
\end{aligned}
$$

$$
\left\{\begin{aligned}
\frac{\partial Q}{\partial t} & =-\vec{\nabla} \cdot(\mathbf{U} Q)-\eta \triangle Q-K Q+H_{c}^{*} V_{2 C}\left(H_{c} C-C_{o b s}\right) \\
\frac{\partial Q}{\partial \vec{n}} & =0 \text { on } S_{W}, \\
\mathbf{U} \vec{n} Q+\eta \frac{\partial Q}{\partial \vec{n}} & =0 \text { on } \Gamma_{2}, \\
Q & =0 \text { on } \Gamma_{1}, \\
Q(T) & =0,
\end{aligned}\right.
$$

$$
\left\{\begin{array}{l}
V_{1 X}\left(U-X_{0}\right)-P(0)=0 \\
V_{1 C}\left(V-C_{0}\right)-Q(0)=0
\end{array}\right.
$$


where $P=\left(P_{1}, P_{2}, P_{3}\right)^{T}$ and $Q$ are the adjoint variables with respect to $X$ and $C$, $F_{0}(Q, C)=\left(0, Q \frac{\partial C}{\partial x}, Q \frac{\partial C}{\partial y}\right)^{T}$, and $A^{*}(X, P), B^{*}(X, P), F^{*}(X, P)$ are defined by the formula:

$$
\left\{\begin{aligned}
& A^{*}(X, P)= {\left[\begin{array}{c}
-g P_{2}-u P_{1} \\
-u P_{2}-h P_{1} \\
-u P_{3}
\end{array}\right] ; B^{*}(X, P)=\left[\begin{array}{c}
-g P_{3}-v P_{1} \\
-v P_{2} \\
-v P_{3}-h P_{1}
\end{array}\right] ; } \\
& F^{*}(X, P)=-\left[\begin{array}{c}
-\frac{4 g u \sqrt{u^{2}+v^{2}}}{3 K_{x}^{2} h^{7 / 3}} P_{2}-\frac{4 g v \sqrt{u^{2}+v^{2}}}{3 K_{y}^{2} h^{7 / 3}} P_{3}+P_{1} \frac{\partial u}{\partial x}+P_{1} \frac{\partial v}{\partial y} \\
g \frac{\left(u^{2}+v^{2}\right)+u^{2}}{K_{x}^{2} h^{4 / 3} \sqrt{u^{2}+v^{2}}}+\frac{\partial u}{\partial x} \\
g \frac{\left(u^{2}+v^{2}\right)+v^{2}}{K_{y}^{2} h^{4 / 3} \sqrt{u^{2}+v^{2}}}+\frac{\partial v}{\partial y}
\end{array}\right] P_{2}+\frac{g u v P_{3}}{K_{y}^{2} h^{4 / 3} \sqrt{u^{2}+v^{2}}}+P_{3} \frac{\partial v}{\partial x}+P_{1} \frac{\partial h}{\partial x} \\
& P_{3}+\frac{g u v P_{2}}{K_{x}^{2} h^{4 / 3} \sqrt{u^{2}+v^{2}}}+P_{2} \frac{\partial u}{\partial y}+P_{1} \frac{\partial h}{\partial y}
\end{aligned}\right] .
$$

\section{Evaluation of SEnsitivities With RESPECT to THE SOURCE}

As in section 2, we will study the sensitivities with respect to the source $S$. Let the response function be defined by

$$
G_{A}(X, C, S)=\int_{0}^{T} \int_{\Omega_{A}} C(x, y, t) d x d y d t
$$

where $\Omega_{A} \subset \Omega$ is the response region, and $C$ depends on $S$ through (7.3).

We consider some direction $s$ in the space of $S$ and then compute the Gateaux derivative of response function $G_{A}$ with respect to this direction. The Gateaux derivative is presented by the following formula:

$$
\hat{G}_{A}(S, s)=\int_{0}^{T} \int_{\Omega_{A}} \hat{C} d x d y d t .
$$

The Gateaux derivative of $\mathrm{P}$ from equation (7.4) is the solution of the following problem:

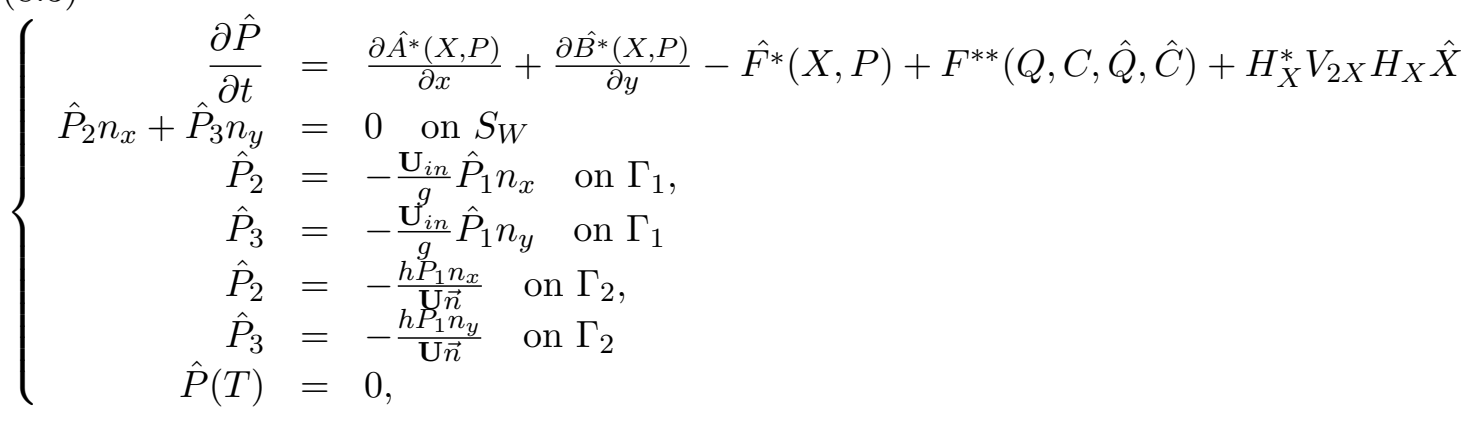

where $F^{* *}(Q, C, \hat{Q}, \hat{C})=\left(0, \hat{Q} \frac{\partial C}{\partial x}+Q \frac{\partial \hat{C}}{\partial x}, \hat{Q} \frac{\partial C}{\partial y}+Q \frac{\partial \hat{C}}{\partial y}\right)^{T}$. Multiplying equation (8.3) by a vector-function $\Psi=\left(\Psi_{1}, \Psi_{2}, \Psi_{3}\right)$ and then integrating in t and over $\Omega$, we have: 


$$
\begin{array}{r}
\int_{0}^{T}\left(\hat{P}, \frac{\partial \Psi}{\partial t}+\frac{\partial A^{* *}(X, \Psi)}{\partial x}+\frac{\partial B^{* *}(X, \Psi)}{\partial y}-F^{* * *}(X, \Psi)\right) d t \\
+\int_{0}^{T}\left(\hat{X}, \frac{\partial A_{X}(X, \Psi)}{\partial x}+\frac{\partial B_{X}(X, \Psi)}{\partial y}-F_{X}(X, \Psi)+H^{*} V_{2 X} H \Psi\right) d t
\end{array}
$$

$=\int_{0}^{T} \int_{\Gamma_{1} \cup \Gamma_{2} \cup S_{W}} \hat{P} E^{* *}(X, \Psi) d\left(\Gamma_{1} \cup \Gamma_{2} \cup S_{W}\right) d t+\int_{0}^{T} \int_{\Gamma_{1} \cup \Gamma_{2} \cup S_{W}} \hat{X} E_{X}(X, \Psi) d\left(\Gamma_{1} \cup \Gamma_{2} \cup S_{W}\right) d t$ $-\int_{0}^{T} \int_{\Omega} \hat{F}^{* *} d \Omega d t-\int_{0}^{T} \int_{\Gamma_{2} \cup S_{W}} \hat{C} Q\left(\Psi_{2} n_{x}+\Psi_{3} n_{y}\right) d\left(\Gamma_{2} \cup S_{W}\right) d t+(\hat{P}(T), \Psi(T))-(\hat{P}(0), \Psi(0))$,

where: $\hat{F}^{* *}=\hat{Q}\left(\Psi_{2} \cdot \frac{\partial C}{\partial x}+\Psi_{3} \cdot \frac{\partial C}{\partial y}\right)-\hat{C} \cdot \frac{\partial Q \Psi_{2}}{\partial x}-\hat{C} \cdot \frac{\partial Q \Psi_{3}}{\partial y}$,

$(8.5)$

$$
\begin{aligned}
& A^{* *}(X, \Psi)=\left[\begin{array}{c}
u \Psi_{1}+h \Psi_{2} \\
g \Psi_{1}+u \Psi_{2} \\
u \Psi_{3}
\end{array}\right] ; \quad B^{* *}(X, \Psi)=\left[\begin{array}{c}
v \Psi_{1}+h \Psi_{3} \\
v \Psi_{2} \\
g \Psi_{1}+v \Psi_{3}
\end{array}\right]
\end{aligned}
$$

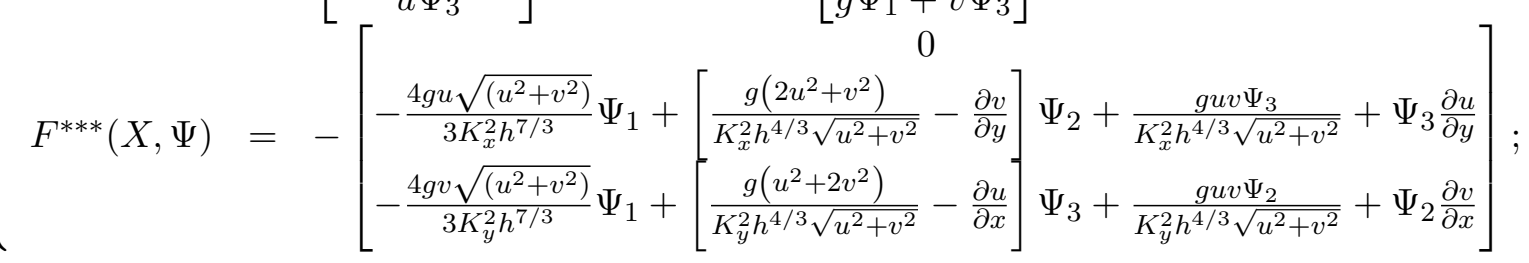

$$
\begin{aligned}
& \left\{\begin{aligned}
A_{X}(X, \Psi)= & {\left[\begin{array}{c}
0 \\
P_{3} \Psi_{3} \\
-P_{3} \Psi_{2}
\end{array}\right] ; B_{X}(X, \Psi)=\left[\begin{array}{c}
0 \\
-P_{2} \Psi_{3} \\
P_{2} \Psi_{2}
\end{array}\right] } \\
F_{X}(X, \Psi)= & -\left[\begin{array}{l}
F_{h}(X, \Psi) \\
F_{u}(X, \Psi) \\
F_{v}(X, \Psi)
\end{array}\right]
\end{aligned}\right. \\
& F_{h}(X, \Psi)=\frac{28 g u \sqrt{u^{2}+v^{2}}}{9 K_{x}^{2} h^{10 / 3}} P_{2} \Psi_{1}+\frac{28 g v \sqrt{u^{2}+v^{2}}}{9 K_{y}^{2} h^{10 / 3}} P_{3} \Psi_{1} \\
& -\frac{4 g\left(2 u^{2}+v^{2}\right)}{3 K_{x}^{2} h^{7 / 3} \sqrt{u^{2}+v^{2}}} P_{2} \Psi_{2}-\frac{4 g\left(u^{2}+2 v^{2}\right)}{3 K_{y}^{2} h^{7 / 3} \sqrt{u^{2}+v^{2}}} P_{3} \Psi_{3} \\
& -\frac{4 g u v}{3 K_{x}^{2} h^{7 / 3} \sqrt{u^{2}+v^{2}}} P_{2} \Psi_{3}-\frac{4 g u v}{3 K_{y}^{2} h^{7 / 3} \sqrt{u^{2}+v^{2}}} P_{3} \Psi_{2}-\frac{\partial P_{1}}{\partial y} \Psi_{3}-\frac{\partial P_{1}}{\partial x} \Psi_{2}, \\
& F_{u}(X, \Psi)=-\frac{4 g\left(2 u^{2}+v^{2}\right)}{3 K_{x}^{2} h^{7 / 3} \sqrt{u^{2}+v^{2}}} P_{2} \Psi_{1}-\frac{4 g u v}{3 K_{y}^{2} h^{7 / 3} \sqrt{u^{2}+v^{2}}} P_{3} \Psi_{1}+\frac{g u\left(2 u^{2}+3 v^{2}\right)}{K_{x}^{2} h^{4 / 3}\left(u^{2}+v^{2}\right)^{3 / 2}} P_{2} \Psi_{2} \\
& +\frac{g u^{3}}{K_{y}^{2} h^{4 / 3}\left(u^{2}+v^{2}\right)^{3 / 2}} P_{3} \Psi_{3}+\frac{g v^{3} P_{2} \Psi_{3}}{K_{x}^{2} h^{4 / 3}\left(u^{2}+v^{2}\right)^{3 / 2}}+\frac{g v^{3} P_{3} \Psi_{2}}{K_{y}^{2} h^{4 / 3}\left(u^{2}+v^{2}\right)^{3 / 2}}-\frac{\partial P_{2}}{\partial x} \Psi_{2}-\frac{\partial P_{1}}{\partial x} \Psi_{1}-\frac{\partial P_{3}}{\partial x} \Psi_{3},
\end{aligned}
$$




$$
\begin{gathered}
F_{v}(X, \Psi)=-\frac{4 g\left(u^{2}+2 v^{2}\right)}{3 K_{y}^{2} h^{7 / 3}} P_{3} \Psi_{1}-\frac{4 g u v}{3 K_{x}^{2} h^{7 / 3} \sqrt{u^{2}+v^{2}}} P_{2} \Psi_{1} \\
+\frac{g v^{3}}{K_{x}^{2} h^{4 / 3}\left(u^{2}+v^{2}\right)^{3 / 2}} P_{2} \Psi_{2}+\frac{g u^{3} P_{3} \Psi_{2}}{K_{y}^{2} h^{4 / 3}\left(u^{2}+v^{2}\right)^{3 / 2}}+\frac{g u^{3} P_{2} \Psi_{3}}{K_{x}^{2} h^{4 / 3}\left(u^{2}+v^{2}\right)^{3 / 2}} \\
+\frac{g v\left(3 u^{2}+2 v^{2}\right) \Psi_{3} P_{3}}{K_{y}^{2} h^{4 / 3}\left(u^{2}+v^{2}\right)^{3 / 2}}-\frac{\partial P_{1}}{\partial y} \Psi_{1}-\frac{\partial P_{2}}{\partial y} \Psi_{2}-\frac{\partial P_{3}}{\partial y} \Psi_{3} \\
E^{* *}(X, \Psi)=\left[\begin{array}{c}
\Psi_{1} \mathbf{U} \vec{n}+h\left(\Psi_{2} n_{x}+\Psi_{3} n_{y}\right) \\
g \Psi_{1} n_{x}+\mathbf{U} \vec{n} \Psi_{2} \\
g \Psi_{1} n_{y}+\mathbf{U} \vec{n} \Psi_{3}
\end{array}\right] ; E_{X}(X, \Psi)=\left[\begin{array}{c}
0 \\
P_{2} \Psi_{3} n_{y}-P_{3} \Psi_{3} n_{x} \\
P_{3} \Psi_{2} n_{x}-P_{2} \Psi_{2} n_{y}
\end{array}\right] .
\end{gathered}
$$

The Gateaux derivative of $Q$ from equation (7.5) is the solution of the following problem:

$$
\left\{\begin{aligned}
\frac{\partial \hat{Q}}{\partial t} & =-\vec{\nabla} \cdot(\mathbf{U} \hat{Q})-\left(\frac{\partial \hat{u} Q}{\partial x}+\frac{\partial \hat{v} Q}{\partial y}\right)-\eta \triangle \hat{Q}-K \hat{Q}+H_{c}^{*} V_{2 C} H_{c} \hat{C} \\
\frac{\partial \hat{Q}}{\partial \vec{n}} & =0 \quad \text { on } S_{W} \\
\mathbf{U} \vec{n} \hat{Q}+\eta \frac{\partial \hat{Q}}{\partial \vec{n}} & =0 \text { on } \Gamma_{2}, \\
\hat{Q} & =0 \text { on } \Gamma_{1}, \\
\hat{Q}(T) & =0 .
\end{aligned}\right.
$$

Multiplying equation (8.7) by function $\Lambda$ and integrating it in t and over $\Omega$, we have:

$$
\begin{array}{r}
\int_{0}^{T}\left(\hat{Q}, \frac{\partial \Lambda}{\partial t}+u \frac{\partial \Lambda}{\partial x}+v \frac{\partial \Lambda}{\partial y}-\eta \Delta \Lambda-K \Lambda\right) d t+\int_{0}^{T}\left(\hat{X}, F 2_{X C}\right) d t \\
=-\int_{0}^{T}\left(\hat{C}, H_{c}^{*} V_{2 C} H_{c} \Lambda\right) d t+(\hat{Q}(T), \Lambda(T))-(\hat{Q}(0), \Lambda(0)) \\
-\eta \int_{0}^{T} \int_{\partial \Gamma_{1}} \Lambda \frac{\partial \hat{Q}}{\partial \vec{n}} d \Gamma_{1} d t+\eta \int_{0}^{T} \int_{\partial\left(\Gamma_{2} \cup S_{W}\right)} \hat{Q} \frac{\partial \Lambda}{\partial \vec{n}} d\left(\Gamma_{2} \cup S_{W}\right) d t \\
+\int_{0}^{T} \int_{\partial \Gamma_{2}} Q \Lambda\left(\hat{u} n_{x}+\hat{v} n_{y}\right) d \Gamma_{2} d t
\end{array}
$$

where: $F 2_{X C}=\left(0, Q \frac{\partial \Lambda}{\partial x}, Q \frac{\partial \Lambda}{\partial y}\right)^{T}$. The Gateaux derivative of $C$ from equation (6.6) is the solution of the problem:

$$
\left\{\begin{aligned}
\frac{\partial \hat{C}}{\partial t} & =-\mathbf{U} \vec{\nabla} \cdot \hat{C}+\eta \triangle \hat{C}+K \hat{C}+s-\left(\hat{u} \frac{\partial C}{\partial x}+\hat{v} \frac{\partial C}{\partial y}\right) \\
\hat{C}_{\left.\right|_{\Gamma_{1}}} & =0 \\
\left.\frac{\partial \hat{C}}{\partial \vec{n}}\right|_{\Gamma_{2} \cup S_{W}} & =0 \\
\hat{C}(0) & =\hat{V} .
\end{aligned}\right.
$$


Multiplying equation (8.8) by function $\Phi$ and integrating in t and $\Omega$, we have:

$$
\begin{array}{r}
\int_{0}^{T}\left(\hat{C},-\frac{\partial \Phi}{\partial t}-\frac{\partial u \Phi}{\partial x}-\frac{\partial v \Phi}{\partial y}-\eta \Delta \Phi-K \Phi\right) d t+\int_{0}^{T}\left(\hat{X}, F 1_{X C}(C, \Lambda)\right) d t \\
=\int_{0}^{T}(s, \Phi) d t-(\hat{C}(T), \Phi(T))+(\hat{C}(0), \Phi(0)) \\
+\eta \int_{0}^{T} \int_{\partial \Gamma_{1}} \Phi \frac{\partial \hat{C}}{\partial \vec{n}} d \Gamma_{1} d t-\eta \int_{0}^{T} \int_{S_{W}} \hat{C} \frac{\partial \Phi}{\partial \vec{n}} d S_{W} d t-\int_{0}^{T} \int_{\partial\left(\Gamma_{2}\right)} \hat{C}\left(\mathbf{U} \vec{n} \Phi+\eta \frac{\partial \Phi}{\partial \vec{n}}\right) d \Gamma_{2} d t
\end{array}
$$

where $F 1_{X C}=\left(0, \Phi \frac{\partial C}{\partial x}, \Phi \frac{\partial C}{\partial y}\right)^{T}$. We denote

$$
F_{X C}=F 2_{X C}+F 1_{X C}=\left[\begin{array}{c}
0 \\
Q \frac{\partial \Lambda}{\partial x}+\Phi \frac{\partial C}{\partial x} \\
Q \frac{\partial \Lambda}{\partial y}+\Phi \frac{\partial C}{\partial y}
\end{array}\right] .
$$

The Gateaux derivative of $X$ from equation (6.5) is the solution of the problem:

$$
\left\{\begin{aligned}
\frac{\partial \hat{X}}{\partial t}+\frac{\partial \hat{\mathbf{A}}(X)}{\partial x}+\frac{\partial \hat{\mathbf{B}}(X)}{\partial y} & =\hat{F}(X), \quad \text { in } \Omega, \\
n_{x} \hat{u}+n_{y} \hat{v} & =0, \quad \text { on } \Gamma_{1} \bigcup S_{W}, \\
\hat{h} & =0, \quad \text { on } \Gamma_{2}, \\
\hat{X}(0) & =\hat{U} .
\end{aligned}\right.
$$

Multiplying equation (8.10) by a vector-function $P^{1}=\left(P_{1}^{1}, P_{2}^{1}, P_{3}^{1}\right)^{T}$ and integrating in t and $\Omega$, we have:

$$
\begin{array}{r}
\int_{0}^{T}\left(\hat{X}, \frac{\partial P^{1}}{\partial t}-\frac{\partial A^{*}\left(X, P^{1}\right)}{\partial x}-\frac{\partial B^{*}\left(X, P^{1}\right)}{\partial y}+F^{*}\left(X, P^{1}\right)\right) d t= \\
\int_{0}^{T} \int_{\Gamma_{1} \cup \Gamma_{2} \cup S_{W}} \hat{X} E^{*}\left(X, P^{1}\right) d\left(\Gamma_{1} \cup \Gamma_{2} \cup S_{W}\right) d t+\left(\hat{X}(T), P^{1}(T)\right)-\left(\hat{X}(0), P^{1}(0)\right),
\end{array}
$$

where the vector-functions $A^{*}\left(X, P^{1}\right), B^{*}\left(X, P^{1}\right), F^{*}\left(X, P^{1}\right)$ are shown in formula (7.7) with variables $X, P^{1}$, and

$$
E^{*}\left(X, P^{1}\right)=\left[\begin{array}{c}
\mathbf{U} \vec{n} P_{1}^{1}+g\left(P_{2}^{1} n_{x}+P_{3}^{1} n_{y}\right) \\
\mathbf{U} \vec{n} P_{2}^{1}+h P_{1}^{1} n_{x} \\
\mathbf{U} \vec{n} P_{3}^{1}+h P_{1}^{1} n_{y}
\end{array}\right] .
$$

The Gateaux derivatives of $U$ and $V$ from equation (7.6) are the solutions of the equations:

$$
V_{1 X} \hat{U}-\hat{P}(0)=0, \quad V_{1 C} \hat{V}-\hat{Q}(0)=0 .
$$

Multiplying the first equation in (8.12) by $\psi=\left(\psi_{1}, \psi_{2}, \psi_{3}\right)$, and the second one by $\phi$, integrating them over $\Omega$, we have:

$$
\int_{\Omega} V_{1 C} \hat{V} \phi d \Omega-\int_{\Omega} \hat{Q}(0) \phi d \Omega=0, \quad \int_{\Omega} V_{1 X} \hat{U} \psi d \Omega-\int_{\Omega} \hat{P}(0) \psi d \Omega=0 .
$$


Adding the obtained integral equalities (8.4)-(8.13), we have:

$$
\begin{aligned}
& \int_{0}^{T}\left(\hat{C},-\frac{\partial \Phi}{\partial t}-\frac{\partial u \Phi}{\partial x}-\frac{\partial v \Phi}{\partial y}-\eta \Delta \Phi-K \Phi-\frac{\partial Q \Psi_{2}}{d x}-\frac{\partial Q \Psi_{3}}{d y}+H_{c}^{*} V_{2 C} H_{c} \Lambda\right) d t \\
& +\int_{0}^{T}\left(\hat{P}, \frac{\partial \Psi}{\partial t}+\frac{\partial A^{* *}(X, \Psi)}{\partial x}+\frac{\partial B^{* *}(X \Psi)}{\partial y}-F^{* * *}(X, \Psi)\right) d t \\
& +\int_{0}^{T}\left(\hat{Q}, \frac{\partial \Lambda}{\partial t}+u \frac{\partial \Lambda}{\partial x}+v \frac{\partial \Lambda}{\partial y}-\eta \Delta \Lambda-K \Lambda+\Psi_{2} \frac{\partial C}{\partial x}+\Psi_{3} \frac{\partial C}{\partial y}\right) d t \\
& +\int_{\Omega}\left(\hat{V}, V_{1 C} \phi-\Phi(0)\right) d \Omega+\int_{\Omega}\left(\hat{U}, V_{1 X} \psi-P^{1}(0)\right) d \Omega \\
& +\int_{0}^{T}\left(\hat{X}, \frac{\partial A_{X}(X, \Psi)}{\partial x}+\frac{\partial B_{X}(X, \Psi)}{\partial y}-F_{X}(X, \Psi)+H_{X}^{*} V_{2 X} H_{X} \Psi+F_{X C}\right) d t \\
& -\int_{0}^{T}\left(\hat{X}, \frac{\partial P^{1}}{\partial t}-\frac{\partial A^{*}\left(X, P^{1}\right)}{\partial x}-\frac{\partial B^{*}\left(X, P^{1}\right)}{\partial y}+F^{*}\left(X, P^{1}\right)\right) d t \\
& =\int_{0}^{T}(s, \Phi) d t-(\hat{P}(0), \Psi(0)-\psi)-(\hat{Q}(0), \Lambda(0)-\phi)+\int_{0}^{T} \int_{\Gamma_{1} \cup \Gamma_{2} \cup S_{W}} \hat{P} E^{* *}(X, \Psi) d\left(\Gamma_{1} \cup \Gamma_{2} \cup S_{W}\right) d t \\
& -\eta \int_{0}^{T} \int_{\partial \Gamma_{1}} \Lambda \frac{\partial \hat{Q}}{\partial \vec{n}} d \Gamma_{1} d t+\eta \int_{0}^{T} \int_{\partial\left(\Gamma_{2} \cup S_{W}\right)} \hat{Q} \frac{\partial \Lambda}{\partial \vec{n}} d\left(\Gamma_{2} \cup S_{W}\right) d t \\
& -\int_{0}^{T} \int_{\partial \Gamma_{1}} \Phi \frac{\partial \hat{C}}{\partial \vec{n}} d \Gamma_{1} d t+\int_{0}^{T} \int_{S_{W}} \hat{C}\left(\eta \frac{\partial \Phi}{\partial \vec{n}}-Q\left(\Psi_{2} n_{x}+\Psi_{3} n_{y}\right)\right) d S_{W} d t \\
& -\int_{0}^{T} \int_{\partial\left(\Gamma_{2}\right)} \hat{C}\left(\mathbf{U} \vec{n} \Phi+\eta \frac{\partial \Phi}{\partial \vec{n}}+Q\left(\Psi_{2} n_{x}+\Psi_{3} n_{y}\right)\right) d \Gamma_{2} d t+\int_{0}^{T} \int_{\partial \Gamma_{2}} Q \Lambda\left(\hat{u} n_{x}+\hat{v} n_{y}\right) d \Gamma_{2} d t \\
& +\int_{0}^{T} \int_{\Gamma_{1} \cup \Gamma_{2} \cup S_{W}}\left(\hat{X}, E_{X}(X, \Psi)-E^{*}\left(X, P^{1}\right)\right) d\left(\Gamma_{1} \cup \Gamma_{2} \cup S_{W}\right) d t \\
& V_{1 C} \Lambda(0)=\Phi(0), \quad V_{1 X} \Psi(0)=P^{1}(0) .
\end{aligned}
$$


If $P^{1}, \Psi, \Lambda, \Phi$ are the solutions of the following problems:

$(8.16)$

$$
\left\{\begin{aligned}
-\frac{\partial P^{1}}{\partial t}+\frac{\partial\left(A^{*}\left(X, P^{1}\right)+A_{X}(X, \Psi)\right)}{\partial x}+\frac{\partial\left(B^{*}\left(X, P^{1}\right)+B_{X}(X, \Psi)\right)}{\partial y}- & \\
-F^{*}\left(X, P^{1}\right)-F_{X}(X, \Psi)+H_{X}^{*} V_{2 X} H_{X} \Psi+F_{X C}(C, \Lambda) & =0 \\
P_{2}^{1} n_{x}+P_{3}^{1} n_{y} & =0 \text { on } S_{W} \\
P_{2}^{1} & =-\frac{h P_{1}^{1} n_{x}}{\mathbf{U} \vec{n}} \text { on } \Gamma_{2}, \\
P_{3}^{1} & =-\frac{h P_{1}^{1} n_{y}}{\mathbf{U} \vec{n}} \text { on } \Gamma_{2} \\
P_{2}^{1} & =-\frac{\mathbf{U}_{i n}}{g} P_{1}^{1} n_{x} \text { on } \Gamma_{1}, \\
P_{3}^{1} & =-\frac{\mathbf{U}_{i n}}{g} P_{1}^{1} n_{y} \text { on } \Gamma_{1} \\
P^{1}(T) & =0,
\end{aligned}\right.
$$

(8.17)

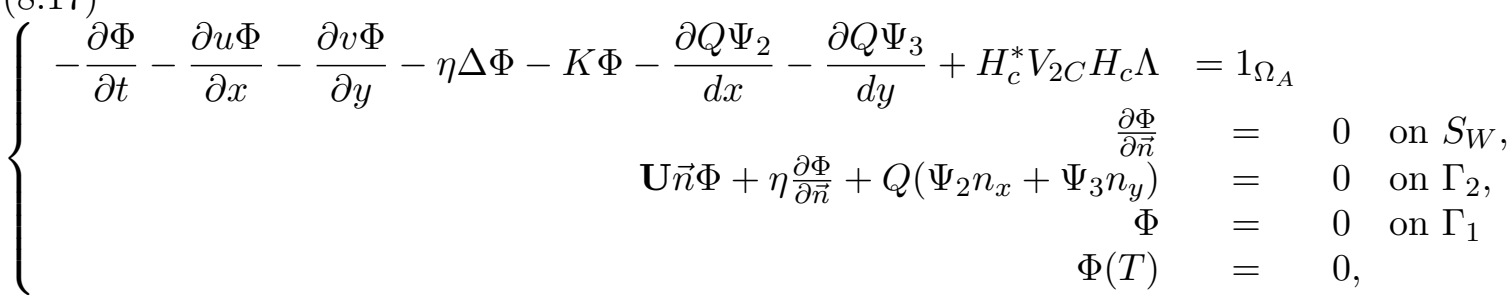

$$
\begin{gathered}
\left\{\begin{aligned}
\frac{\partial \Psi}{\partial t} & =-\frac{\partial A^{* *}(X, \Psi)}{\partial x}-\frac{\partial B^{* *}(X, \Psi)}{\partial y}+F^{* * *}(X, \Psi) \\
\Psi_{2} n_{x}+\Psi_{3} n_{y} & =0 \text { on } S_{W} \cup \Gamma_{1}, \\
\Psi_{1} & =0 \text { on } \Gamma_{2} \\
V_{1 X} \Psi(0) & =P^{1}(0)
\end{aligned}\right. \\
\left\{\begin{aligned}
\frac{\partial \Lambda}{\partial t}+u \frac{\partial \Lambda}{\partial x}+v \frac{\partial \Lambda}{\partial y}-\eta \Delta \Lambda-K \Lambda+\Psi_{2} \frac{\partial C}{\partial x}+\Psi_{3} \frac{\partial C}{\partial y} & =0 \\
\Lambda & =0 \text { on } \Gamma_{1}, \\
\frac{\partial \Lambda}{\partial \vec{n}} & =0 \text { on } S_{W} \cup \Gamma_{2}, \\
V_{1 C} \Lambda(0) & =\Phi(0)
\end{aligned}\right.
\end{gathered}
$$

using equations (8.2), (8.14), the gradient of response function $G_{A}$ is calculated by the formula:

$$
\hat{G}_{A}(S, s)=\int_{0}^{T}\left(1_{\Omega}, \hat{C}\right) d t=\int_{0}^{T}(s, \Phi) d t
$$

Hence

$$
\frac{d G}{d S}=\int_{0}^{T} \Phi d t \quad \text { in } \Omega
$$




\section{Non-STANDARD PROBLEM}

By the way shown in section 3 we will solve the system (8.16)-(8.19). Instead of (8.18), (8.19) we consider the problems:

$$
\begin{gathered}
\left\{\begin{aligned}
\frac{\partial \Psi}{\partial t} & =-\frac{\partial A^{* *}(X, \Psi)}{\partial x}-\frac{\partial B^{* *}(X, \Psi)}{\partial y}+F^{* * *}(X, \Psi) \\
\Psi_{2} n_{x}+\Psi_{3} n_{y} & =0 \text { on } S_{W} \\
\Psi_{2} n_{x}+\Psi_{3} n_{y} & =0 \text { on } \Gamma_{1}, \\
\Psi_{1} & =0 \text { on } \Gamma_{2}, \\
\Psi(0) & =v_{1},
\end{aligned}\right. \\
\left\{\begin{aligned}
\frac{\partial \Lambda}{\partial t}+u \frac{\partial \Lambda}{\partial x}+v \frac{\partial \Lambda}{\partial y}-\eta \Delta \Lambda-K \Lambda+\Psi_{2} \frac{\partial C}{\partial x}+\Psi_{3} \frac{\partial C}{\partial y} & =0 \\
\Lambda & =0 \text { on } \Gamma_{1}, \\
\frac{\partial \Lambda}{\partial \vec{n}} & =0 \text { on } \Gamma_{2} \cup S_{W}, \\
\Lambda(0) & =v_{2} .
\end{aligned}\right.
\end{gathered}
$$

We assume that for given $v=\left(v_{1}, v_{2}\right) \in X_{X} \times X_{C}$ the problems (8.16), (8.17), (9.1),(9.2) have the unique solution $P^{1}, \Phi, \Psi, \Lambda$ for $t \in[0, T]$. We define the cost function:

$$
J_{(\Psi, \Phi)}(v)=\frac{1}{2}\left\|V_{1 X} v_{1}-P_{1}\left(0, v_{1}\right)\right\|_{X_{X}}^{2}+\frac{1}{2}\left\|V_{1 C} v_{2}-\Phi\left(0, v_{2}\right)\right\|_{X_{C}}^{2} .
$$

Minimizing $J_{(\Psi, \Phi)}$ we have the value $v^{*}=\left(v_{1}^{*}, v_{2}^{*}\right)$. If at the optimum the equations

$$
V_{1 X} v_{1}-P_{1}\left(0, v_{1}\right)=0, \quad V_{1 C} v_{2}-\Phi\left(0, v_{2}\right)=0
$$

are satisfied, then the problem will be solved.

Following the reasoning of section 3 , we can obtain the gradient of $J_{(\Psi, \Phi)}$ through the adjoint variables. Let $R^{1}, R^{2}, Q_{1}$ and $Q_{2}$ satisfy the following problems:

$$
\begin{aligned}
& \left\{\begin{aligned}
\frac{\partial R^{1}}{\partial t}+\frac{\partial A^{* *}\left(X, R^{1}\right)}{\partial x}+\frac{\partial B^{* *}\left(X, R^{1}\right)}{\partial y}-F^{* * *}\left(X, R^{1}\right) & =0 \\
R_{2}^{1} n_{x}+R_{3}^{1} n_{y} & =0 \text { on } S_{W} \cup \Gamma_{1}, \\
R_{1}^{1} & =0 \text { on } \Gamma_{2}, \\
R^{1}(0) & =V_{1 X} v_{1}-P^{1}(0),
\end{aligned}\right. \\
& \left\{\begin{aligned}
-\frac{\partial R^{2}}{\partial t}+\frac{\partial A_{R^{2}}}{\partial x}+\frac{\partial B_{R^{2}}}{\partial y}-F_{R^{2}}+H^{*} V_{2 X} H R^{1}=0 & \\
R_{2}^{2} n_{x}+R_{3}^{2} n_{y} & =0 \text { on } S_{W} \\
R_{2}^{2} & =-\frac{\left(h R_{1}^{2}+Q Q_{1}\right) n_{x}}{\mathbf{U} \vec{n}} \quad \text { on } \Gamma_{2}, \\
R_{3}^{2} & =-\frac{\left(h R_{1}^{2}+Q Q_{1}\right) n_{y}}{\mathbf{U} \vec{n}} \text { on } \Gamma_{2}, \\
R_{2}^{2} & =-\frac{\mathbf{U} \vec{n} R_{1}^{2} n_{x}}{\mathbf{g} \overrightarrow{n_{2}^{2}}} \text { on } \Gamma_{1}, \\
R_{3}^{2} & =-\frac{\mathbf{U} R_{1}^{2} n_{y}}{g} \text { on } \Gamma_{1}, \\
R^{2}(T) & =0,
\end{aligned}\right.
\end{aligned}
$$




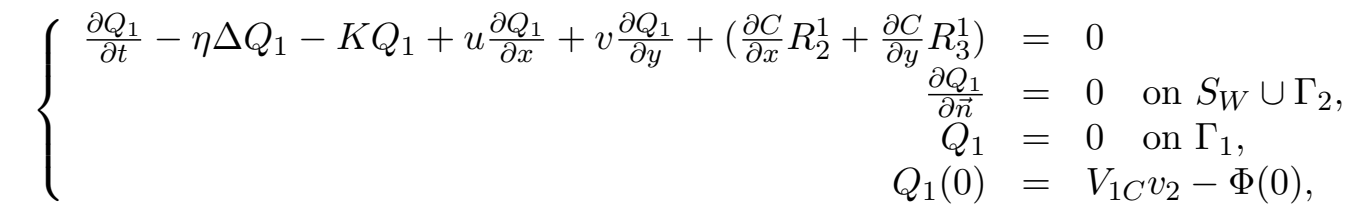

$$
\left\{\begin{aligned}
\frac{\partial Q_{2}}{\partial t}+\frac{\partial u Q_{2}}{\partial x}+\frac{\partial v Q_{2}}{\partial y}+\eta \Delta Q_{2}+K Q_{2}-H_{c}^{*} V_{2 C} H_{c} Q_{1}+\left(\frac{\partial Q R_{2}^{1}}{\partial x}+\frac{\partial Q R_{3}^{1}}{\partial y}\right) & =0 \\
\eta \frac{\partial Q_{2}}{\partial \vec{n}}+\mathbf{U} \vec{n} Q_{2}+Q\left(R_{2}^{1} n_{x}+R_{3}^{1} n_{y}\right) & =0 \text { on } \Gamma_{2}, \\
Q_{2} & =0 \text { on } \Gamma_{1}, \\
\frac{\partial Q_{2}}{\partial \vec{n}} & =0 \text { on } S_{W}, \\
Q_{2}(T) & =0,
\end{aligned}\right.
$$

where the function vectors $A^{* *}\left(X, R^{1}\right), B^{* *}\left(X, R^{1}\right), F^{* * *}\left(X, R^{1}\right)$ are defined by formula (8.5) with variable $R^{1}$ instead of $\Psi$, and

$$
\begin{gathered}
A_{R^{2}}=A^{*}\left(X, R^{2}\right)+A_{X}\left(X, R^{1}\right), \quad B_{R^{2}}=B^{*}\left(X, R^{2}\right)+B_{X}\left(X, R^{1}\right), \\
F_{R^{2}}=F^{*}\left(X, R^{2}\right)+F_{\Psi}-\left[\begin{array}{c}
0 \\
Q \frac{\partial Q_{1}}{\partial x}+Q_{2} \frac{\partial C}{\partial x} \\
Q \frac{\partial Q_{1}}{\partial y}+Q_{2} \frac{\partial C}{\partial y}
\end{array}\right], F_{\Psi}=-\left(F_{\Psi_{1}}, F_{\Psi_{2}}, F_{\Psi_{3}}\right)^{T} \\
F_{\Psi_{1}}=\frac{28 g u \sqrt{u^{2}+v^{2}}}{9 K_{x}^{2} h^{10 / 3}} P_{2} R_{1}^{1}+\frac{28 g v \sqrt{u^{2}+v^{2}}}{9 K_{y}^{2} h^{10 / 3}} P_{3} R_{1}^{1} \\
-\frac{4 g\left(2 u^{2}+v^{2}\right)}{3 K_{x}^{2} h^{7 / 3} \sqrt{u^{2}+v^{2}}} P_{2} R_{2}^{1}-\frac{4 g u v}{3 K_{y}^{2} h^{7 / 3} \sqrt{u^{2}+v^{2}}} P_{3} R_{2}^{1} \\
-\frac{4 g u v}{3 K_{x}^{2} h^{7 / 3} \sqrt{u^{2}+v^{2}}} P_{2} R_{3}^{1}-\frac{4 g\left(u^{2}+2 v^{2}\right)}{3 K_{y}^{2} h^{7 / 3} \sqrt{u^{2}+v^{2}}} P_{3} R_{3}^{1}-\frac{\partial P_{1}}{\partial x} R_{2}^{1}-\frac{\partial P_{1}}{\partial y} R_{3}^{1}
\end{gathered}
$$

$$
\begin{array}{r}
F_{\Psi_{2}}=-\frac{4 g\left(2 u^{2}+v^{2}\right)}{3 K_{x}^{2} h^{7 / 3} \sqrt{u^{2}+v^{2}}} P_{2} R_{1}^{1}-\frac{4 g u v}{3 K_{y}^{2} h^{7 / 3} \sqrt{u^{2}+v^{2}}} P_{3} R_{1}^{1}+\frac{g u\left(2 u^{2}+3 v^{2}\right)}{K_{x}^{2} h^{4 / 3}\left(u^{2}+v^{2}\right)^{3 / 2}} P_{2} R_{2}^{1} \\
+\frac{g v^{3}}{K_{y}^{2} h^{4 / 3}\left(u^{2}+v^{2}\right)^{3 / 2}} P_{3} R_{2}^{1}+\frac{g v^{3}}{K_{x}^{2} h^{4 / 3}\left(u^{2}+v^{2}\right)^{3 / 2}} P_{2} R_{3}^{1}+\frac{g u^{3}}{K_{y}^{2} h^{4 / 3}\left(u^{2}+v^{2}\right)^{3 / 2}} P_{3} R_{3}^{1} \\
-\frac{\partial P_{1}}{\partial x} R_{1}^{1}-\frac{\partial P_{2}}{\partial x} R_{2}^{1}-\frac{\partial P_{3}}{\partial x} R_{3}^{1},
\end{array}
$$

$$
\begin{array}{r}
F_{\Psi_{3}}=-\frac{4 g\left(u^{2}+2 v^{2}\right)}{3 K_{y}^{2} h^{7 / 3} \sqrt{u^{2}+v^{2}}} P_{3} R_{1}^{1}-\frac{4 g u v}{3 K_{x}^{2} h^{7 / 3} \sqrt{u^{2}+v^{2}}} P_{2} R_{1}^{1}+\frac{g u^{3}}{K_{y}^{2} h^{4 / 3}\left(u^{2}+v^{2}\right)^{3 / 2}} P_{3} R_{2}^{1} \\
+\frac{g v^{3}}{K_{x}^{2} h^{4 / 3}\left(u^{2}+v^{2}\right)^{3 / 2}} P_{2} R_{2}^{1}+\frac{g u^{3}}{K_{x}^{2} h^{4 / 3}\left(u^{2}+v^{2}\right)^{3 / 2}} P_{2} R_{3}^{1}+\frac{g v\left(3 u^{2}+2 v^{2}\right)}{K_{y}^{2} h^{4 / 3}\left(u^{2}+v^{2}\right)^{3 / 2}} P_{3} R_{3}^{1} \\
-\frac{\partial P_{1}}{\partial y} R_{1}^{1}-\frac{\partial P_{2}}{\partial y} R_{2}^{1}-\frac{\partial P_{3}}{\partial y} R_{3}^{1} .
\end{array}
$$

Then, we have the gradient of the cost function $J_{(\Psi, \Phi)}(v)$ :

$$
\nabla J_{(\Psi, \Phi)}(v)=\left(V_{1 X}\left(V_{1 X} v_{1}-P^{1}(0)\right)-R^{2}(0), V_{1 C}\left(V_{1 C} v_{2}-\Phi(0)\right)-Q_{2}(0)\right) .
$$


9.1. Algorithm to calculate the gradient of the response function $G_{A}$. We have the following algorithm to calculate the gradient of the response function $G_{A}$ :

- 1. Solve equations (8.16), (8.17), (9.1), (9.2), (9.5)-(9.8);

- 2. Have $\nabla J_{(\Psi, \Phi)}(v)$ by $(9.13)$;

- 3. Solve the optimal control problem finding the mimimum of $J_{(\Psi, \Phi)}(v)$ with the value $v^{*}=\left(v_{1}^{*}, v_{2}^{*}\right)$

- 4. Put the obtained $v_{1}^{*}, v_{2}^{*}$ into the relation $\Psi(0)=v_{1}^{*}, \Lambda(0)=v_{2}^{*}$;

- 5. Solve again the problems (9.1), (9.2), (8.17);

- 6. Have the value of the gradient of the response function $\frac{d G}{d S}$ by formula (8.20).

\section{Simulation EXPERIMENT ON COMPUting THE RESPONSE-FUnCtion GRADIENT FOR 2D WATER POLLUTION MODEL}

In order to numerically solve the above model equations, a cell-centered finite volume method is used (see [23]), accompanied by an explicit scheme in time [25]. To study the response function's gradient we consider the problem of water flow running into the channel with the lenght $3000 \mathrm{~m}$, the width $800 \mathrm{~m}$, and the bottom elevation $z_{b}=0$. Then the flow domain $\Omega$ is defined by the rectangular area $3000 \mathrm{~m} \times 800 \mathrm{~m}$. The other data of this problem are described in Table 1. In this problem the gate-into channel denoted by $\Gamma_{1}$ is on the place where $x=0, y \in[0,200]$, and the gate out of the channel $\Gamma_{2}$ is on the other place where $x=3000, y \in[600,800]$. The boundary conditions on the in-gate $\Gamma_{1}$ into the channel are: $\left.C\right|_{\Gamma_{1}}=24 \mathrm{mg} / \mathrm{l}$ and $\left.\mathbf{U} \vec{n}\right|_{\Gamma_{1}}=\left.\left(u n_{x}+v n_{y}\right)\right|_{\Gamma_{1}}=0.35 \mathrm{~m} / \mathrm{s}$. The boundary conditions on the solid boundary $S_{W}$ are: $\left.\frac{\partial C}{\partial n}\right|_{S_{W}}=0$ and $\left.\mathbf{U} \vec{n}\right|_{S_{W}}=\left.\left(u n_{x}+v n_{y}\right)\right|_{S_{W}}=0$. The boundary conditions on the out-gate $\Gamma_{2}$ of the channel are $\left.\frac{\partial C}{\partial n}\right|_{\Gamma_{2}}=0$ and $\left.h\right|_{\Gamma_{2}}=7 \mathrm{~m}$. The initial conditions are $u(x, y, 0)=0, v(x, y, 0)=0, h(x, y, 0)=7 \mathrm{~m}$ and $C(x, y, 0)=24$ $\mathrm{mg} / \mathrm{l}$.

\begin{tabular}{|c|c|c|c|c|}
\hline$K_{x}, K_{y}$ & Mesh type & $\eta$ & $K$ & Time step (s) \\
\hline 30.6 & Triangular & $1.7 e^{-6}$ & $-4.05 E^{-6}$ & 1 \\
\hline
\end{tabular}

TABLE 1. Data of the channel

- We will test the problem by considering a "twin-experiment".

- A run of the model (with arbitrary initial values) simulating the true pollutant concentration levels, is used as a reference. The reference run is used to extract the "pseudo" observations, at certain points of the channel. The measurement $X_{0}, C_{0}$ are obtained by the values of $X, C$ at the moment 2000 s of the reference model. The model is running more $100 \mathrm{~s}$, then we have $X_{o b s}$ and $C_{o b s}$ in every time step.

- In the testing model the initial value for the $X(0)=U$ and $C(0)=V$ is taken as the average of other long model runs $(2000 \mathrm{~s})$, with a different initial value. (Note that the initial value for this run does not matter much since we have taken the average over a very long run). The model is running more 100s, 
then $X, C, P, Q$ are obtained by the optimal process (7.3)-(7.6). Then for this time period 100 s the vector functions $\Phi, \Psi, \Lambda, Q_{1}, Q_{2}, R^{1}, R^{2}$ are received by finding the minimum of the cost function $J_{(\Psi, \Phi)}(v)$ using the subsection 9.1 (steps 1-5). Therefore, the gradient of the response function $G$ is obtained by formula (8.20).

- When there is not yet any pollution source put in the middle of the channel with unstructured net, the concentration and velocity fields at one moment are shown in figure 1. The substance comes into the channel by the gate $\Gamma_{1}$ and then the concentration distribution is shown in this figure. Let the model run until the moment 2000s, then we put 1 or 2 sources with the concentration $40 \mathrm{mg} / \mathrm{l}$ in the channel and let the model run more 100s (see figure 2). It is shown that in the cases when the response regions are located in the source places the relative gradient values of response functions and the red areas are larger than the others when the response regions are located far from the source place (see figures 3-4). In the case when the response area is in the middle place between 2 sources the red and green areas are closer to the source places; when response areas are nearby the source place the relative gradient values are larger than the others when the response area are located far from the source places (see figures 3-4).
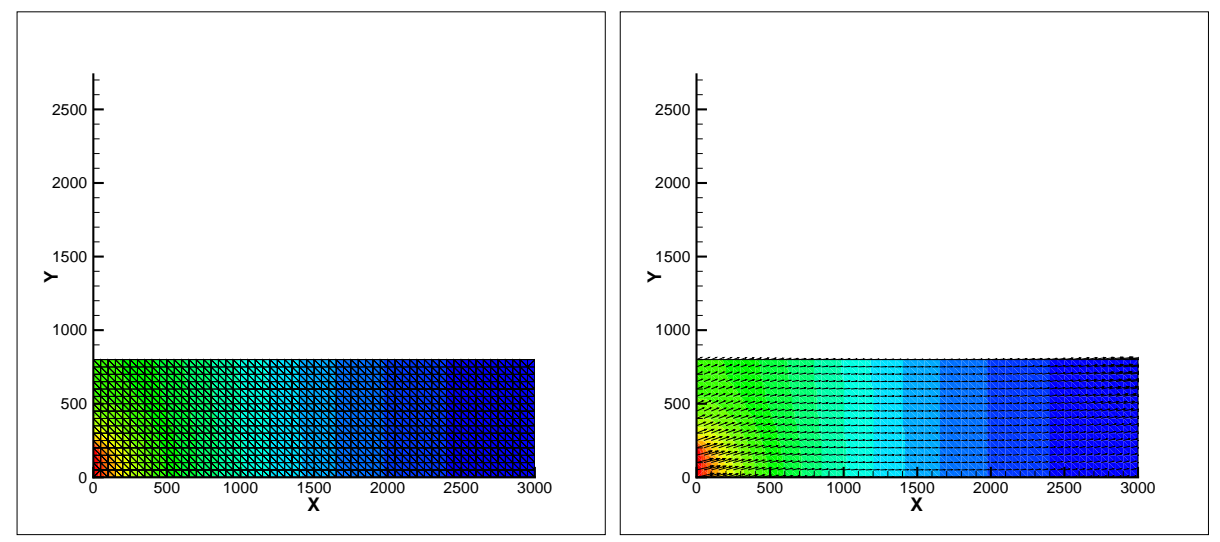

FiguRE 1. Unstructured net with triangular cells before putting the pollution source into the middle of the channel (Left); Velocity field before putting the pollution source into the middle of the channel (Right)

\section{ACKNOWLEDGEMENTS}

The authors acknowledge the support by Region Rhone-Alpes, the MOISE Project, Ambassade de France in Moscow, the Russian Foundation for Basic Research (project 12-01-00322), Russian Federal Research Program 'Kadry', the NAFOSTED's funds and KHCN-TB/13-18 program. 

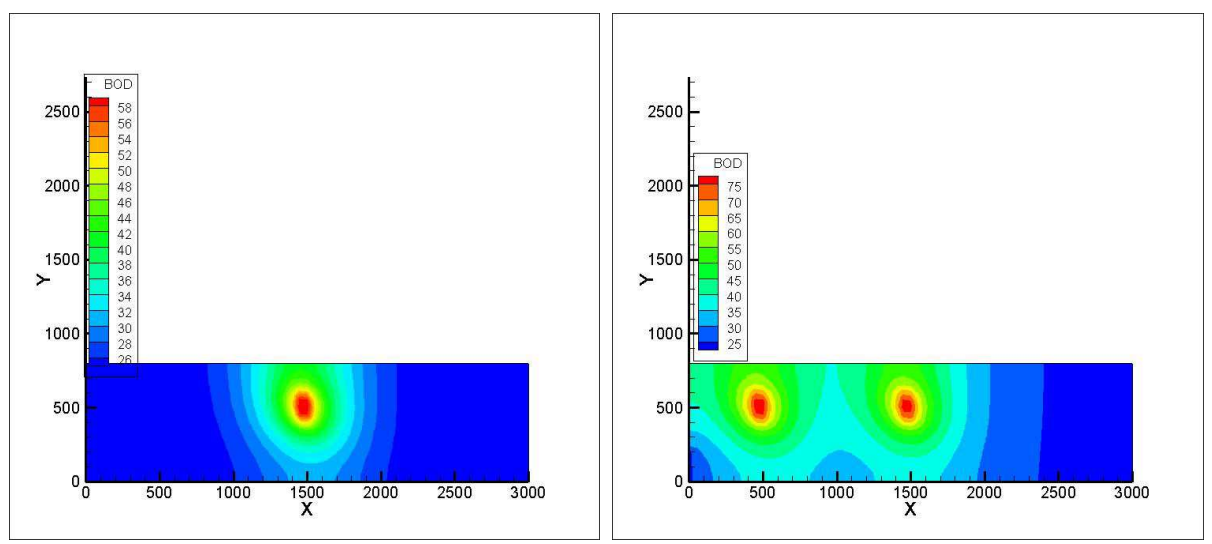

FiguRE 2. Concentration picture after putting 1 pollution source into the channel (Left); Concentration picture after putting 2 pollution sources into the channel (Right)
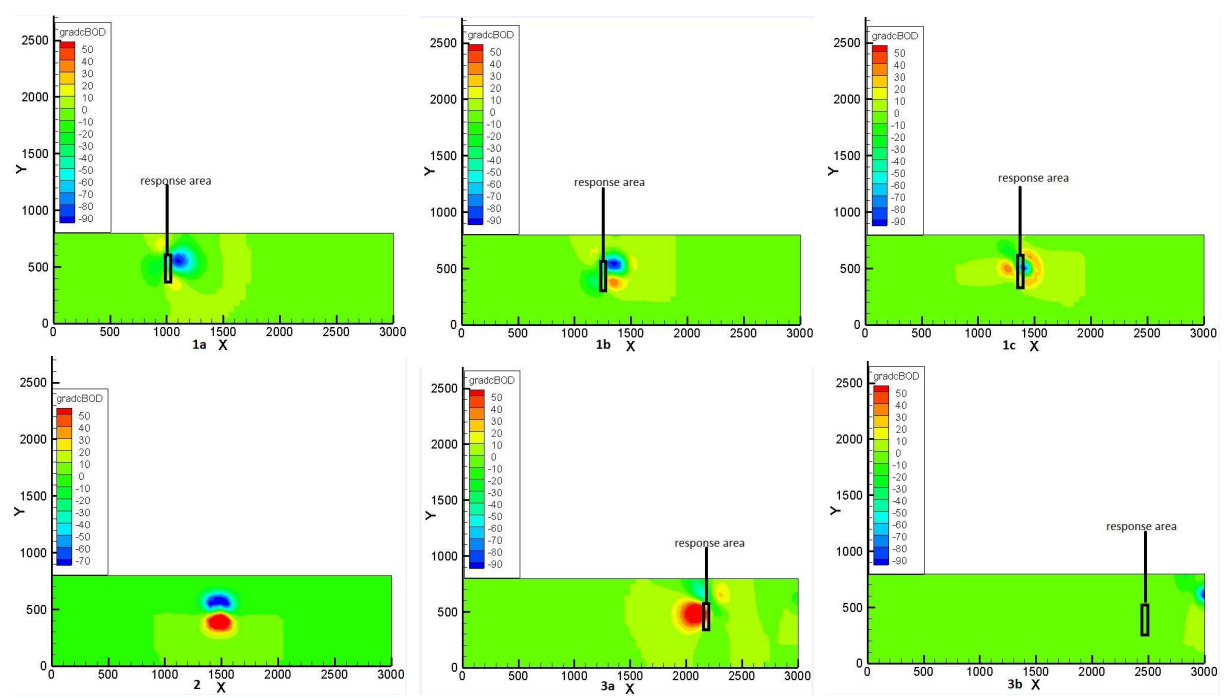

Figure 3. One source in the channel - Relative gradients of the response function in 6 cases of response region places (from left to right) : Response region in the left-hand place of the source region (fig.1a-1c); Response region in the place of the source region ( fig.2); Response regions in the right-hand and far right-hand places of the source region (fig.3a-3b)

\section{REFERENCES}

[1] Cacuci D.G. Sensitivity theory for nonlinear systems: II.Extensions to additional classes of responses. J. Math. Phys, 1981, v.22, pp.2803-2812. 

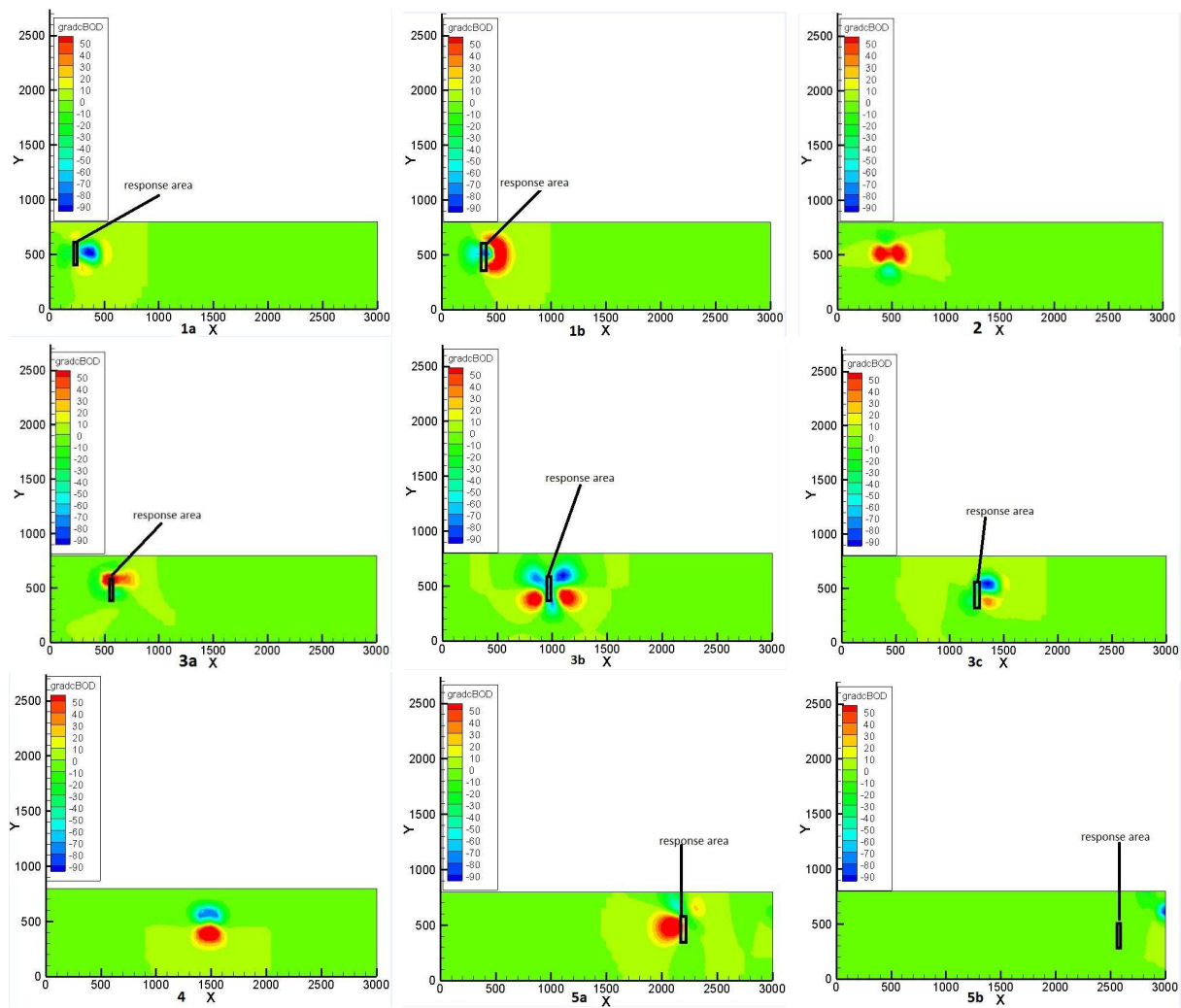

Figure 4. Two sources in the channel - Relative gradients of the response function in 9 cases of the response region places (from left to right) : Response region in the left-hand place of the source regions (fig.1a-1b); Response region in the place of the first source region (fig.2); Response region in the right-hand place nearby the first source region (fig.3a); Response region in the middle between 2 sources (fig. 3b); Response region in the left-hand place nearby the second source region (fig.3c); Response region in the place of the second source region (fig.4); Response region in the right-hand place of the source region (fig.5a-5b)

[2] Chavent G. Local stability of the output least square parameter estimation technique. Math. Appl. Comp., 1983, v.2, pp.3-22.

[3] Dontchev A.L. Perturbations, Approximations and Sensitivity Analysis of Optimal Control Systems.(Lecture Notes in Control and Information Sciences; 52). - Berlin: Springer, 1983.

[4] Gejadze I., Le Dimet F.-X., Shutyaev V., On analysis error covariances in variational data assimilation. SIAM J. Sci. Computing, 2008, v.30, no.4, pp.1847-1874.

[5] Gejadze I., Le Dimet F.-X., Shutyaev V., On optimal solution error covariances in variational data assimilation problems. Journal of Computational Physics, 2010, v.229, pp.2159-2178. 
[6] Gejadze I.Yu., Copeland G.J.M., Le Dimet F.-X., Shutyaev V., Computation of the analysis error covariance in variational data assimilation problems with nonlinear dynamics. Journal of Computational Physics, 2011, v.230, pp.7923-7943.

[7] Glowinski R., Lions J.L. Exact and approximate controllability for distributed parameter systems. Acta Numerica, 1994, v.1, p. 269.

[8] Le Dimet F.-X., Navon I.M., Daescu D.N. Second-order information in data assimilation. Monthly Weather Review, 2002, v.130, no.3, pp.629-648.

[9] LeDimet F.-X., Ngnepieba P., Shutyaev V. On error analysis in data assimilation problems. Russ. J. Numer. Anal. Math. Modelling, 2002, 17, pp.71-97.

[10] LeDimet F.-X., Ngodock, Luong B., Verron J. Sensitivity analysis in variational data assimilation. $J$. Meteorol. Soc. Japan, 1997, v.75 (1B), pp.245-255.

[11] Le Dimet F.X., Talagrand O. Variational algorithms for analysis and assimilation of meteorological observations: theoretical aspects. Tellus, 1986, v.38A, pp.97-110.

[12] Le Dimet F.-X., Shutyaev V. On deterministic error analysis in variational data assimilation. Nonlinear Processes in Geophysics, 2005, 14, p. 1-10.

[13] Lions J.L. Contrôle optimal des systèmes gouvernés par des équations aux dérivées partielles. - Paris: Dunod, 1968.

[14] Lions J.L. Contrôlabilité Exacte Perturbations et Stabilisation de Systèmes Distribués. - Paris: Masson, 1988.

[15] Marchuk G.I., Penenko V.V. Application of optimization methods to the problem of mathematical simulation of atmospheric processes and environment. Modelling and Optimization of Complex Systems: Proc. of the IFIP-TC7 Working conf. - New York: Springer, 1978, pp. 240-252.

[16] Marchuk G.I., Agoshkov V.I., Shutyaev V.P. Adjoint Equations and Perturbation Algorithms in Nonlinear Problems. - New York: CRC Press Inc., 1996.

[17] Navon I.M. Variational data assimilation, optimal parameter estimation and sensitivity analysis for environmental problems. Computational Mechanics'95. - New York: Springer, 1995, v.1, pp.740-746.

[18] Pontryagin L.S., Boltyanskii V.G., Gamkrelidze R.V., Mischenko E.F. The Mathematical Theory of Optimal Processes. - New York: John Wiley, 1962.

[19] Rabier F., Courtier P. Four-dimensional assimilation in the presence of baroclinic instability. Quart. J. Roy. Meteorol. Soc., 1992, v.118, pp.649-672.

[20] Sasaki Y.K. Some basic formalisms in numerical variational analysis. Monthly Weather Review, 1970, v.98, pp.857-883.

[21] Shutyaev V.P. Some properties of the control operator in the problem of data assimilation and iterative algorithms. Russ. J. Numer. Analys. Math. Modelling,1995, v.10, pp.357-371.

[22] Thacker W.C. The role of the Hessian matrix in fitting models to measurements. J. Geophys. Res., 1989, v.94, no.C5, pp.6177-6196.

[23] Sleigh, P.A., Gaskell,P.H., Berzins M. and Wright, N.G. (1998) An unstructured Finite Volume Algorithm for predicting Flow in Rivers and Estuaries, Computers \& Fluids 27, 479-508.

[24] W. Rauch, M. Henze, L. Koncsos, P. Shanahan, L. Somlyody and Vanrolleghem. (1998) River water quality modelling: I. State of the art, Wat. Sci. Tech. , 38, No. 11, 237-244

[25] Tran Thu Ha, Pham Dinh Tuan, Hoang Van Lai, Nguyen Hong Phong (2013) Pollution Estimation based on the 2D Water Transport Model and the Singular Evolutive Interpolated Filter, Submit to Mechanique de Rendu. 